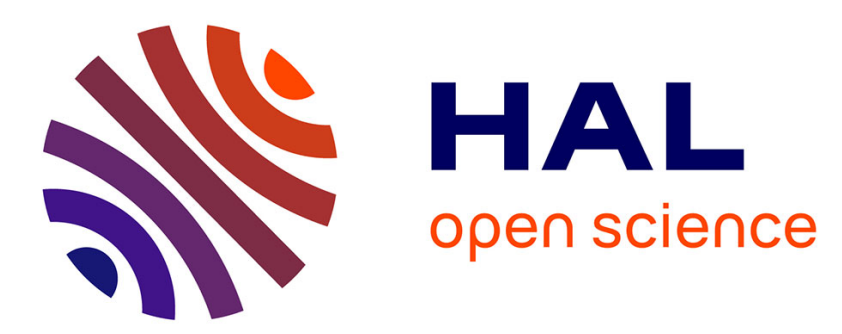

\title{
Chain conformation: A key parameter driving clustering or dispersion in polyelectrolyte - Colloid systems
}

\author{
I. Grillo, I. Morfin, J. Combet
}

\section{To cite this version:}

I. Grillo, I. Morfin, J. Combet. Chain conformation: A key parameter driving clustering or dispersion in polyelectrolyte - Colloid systems. Journal of Colloid and Interface Science, 2020, 561, pp.426-438. 10.1016/j.jcis.2019.11.010 . hal-03059782

\section{HAL Id: hal-03059782 \\ https://hal.science/hal-03059782}

Submitted on 22 Dec 2020

HAL is a multi-disciplinary open access archive for the deposit and dissemination of scientific research documents, whether they are published or not. The documents may come from teaching and research institutions in France or abroad, or from public or private research centers.
L'archive ouverte pluridisciplinaire HAL, est destinée au dépôt et à la diffusion de documents scientifiques de niveau recherche, publiés ou non, émanant des établissements d'enseignement et de recherche français ou étrangers, des laboratoires publics ou privés. 


\section{Chain conformation : a key parameter driving clustering or dispersion in polyelectrolyte - colloid systems.}

Grillo I.(a); Morfin I.(b); Combet J.(c)

(a) Institut Laue Langevin, Large Scale Structures, 71 avenue des Martyrs, CS 20156, 38042 Grenoble Cedex 9, France

(b) Laboratoire interdisciplinaire de physique, CNRS - Université Grenoble Alpes BP 8738402 Saint Martin d'Hères, France

(c) Université de Strasbourg, Institut Charles Sadron, CNRS, UPR 22, 23 rue du Loess, 67034 Strasbourg Cedex 2, France

Graphical Abstract

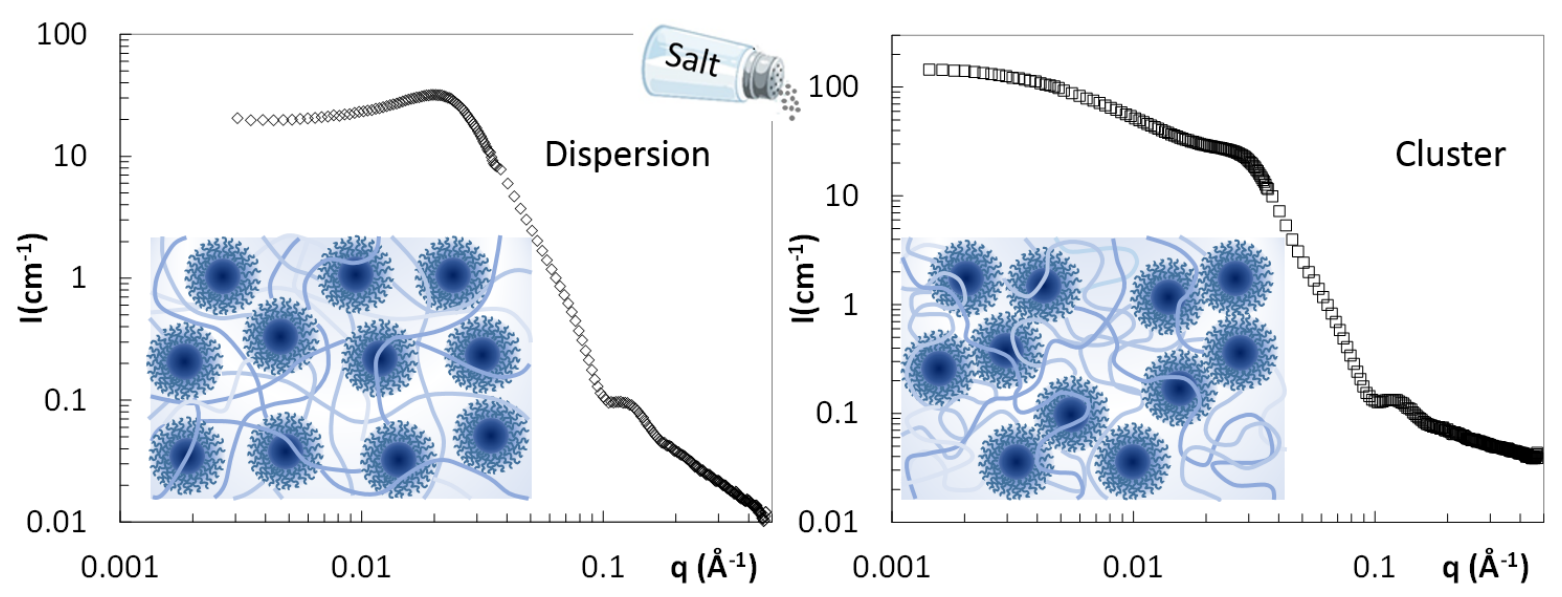

Abstract

The work presents the characterization of Pluronic F127 micellar solutions in presence of hyaluronic acid in semi-dilute regime. The effects of the nature and salt concentration are investigated by differential scanning calorimetry and small angle neutron scattering. Hyaluronic acid reduces the critical micellar temperature to the same extend as an increase of the ionic strength. Within the investigated HA concentration range, the size and shape of the micelles are not modified by the addition of $\mathrm{HA}$ but their dispersion state depends on the salt concentration. By increasing the ionic strength we observe the formation of small micellar clusters which organize into a face-centered cubic liquid crystalline phase at high salt concentration. This behavior is reinforced by increasing the HA concentration. The nature of the salt play also a role and divalent cations such as $\mathrm{Ca}^{2+}$ promote the clustering of micelles and their crystallization. The origin of the aggregative behavior is the change of the HA chain conformation - from stretched to coil- by addition of salt which in turn induces an excluded volume around the micelles and exerts a depletion interaction.

Keywords: Small angle neutron scattering, Pluronic, hyaluronic acid, chain conformation, depletion, ionic strength 


\section{Introduction}

In the medical field and especially for cancer treatment, nanocarrier systems with specific targeting are promising tools to reduce the dose and the side effects, to avoid burst release and prolong the circulation into the blood stream. In addition, they must be easily injectable, biocompatible and biodegradable. Among all the potential carriers, Pluronics copolymers are widely investigated. They are composed by two hydrophilic poly(ethylene oxide) (PEO) groups and a central hydrophobic poly(propylene oxide) (PPO) group. Their self-assembling properties (size and shape of the micelles, critical micellar concentration and temperature) can be varied by changing the EO/PO ratio and group lengths. $\mathrm{F} 127\left(\mathrm{EO}_{100} \mathrm{PO}_{70} \mathrm{EO}_{100}\right)$ used in this work is involved in a considerable number of patents. The high capability of F127 micelles to load hydrophobic molecules, and more specifically drugs is widely recognized [1].

Hyaluronic acid (HA) is a natural linear semirigid polyelectrolyte with a repeating negatively charged disaccharide unit composed of $\mathrm{N}$-acetyl-glucosamine and glucoronic acid. $\mathrm{HA}$ is found in large quantities in animal and human body. It is a common component of synovial fluid and extracellular matrix. However it plays ambiguous roles. Whereas high molecular weight HA (> $500 \mathrm{kDa}$ ) is antiangiogenic, anti-inflammatory and immunosuppressive, low molecular weight (10 - $500 \mathrm{kDa}$ ) is highly angiogenic and pro-inflammatory. $\mathrm{HA}$ is currently investigated as a target-specific material since many malignant cancer cells overexpress HA receptors and its incorporation in nanocarrier systems can be used as targeting ligand [2].

Considering the relevant properties of each of these macromolecules, several studies report on the potential applications of the mixed systems for tissue engineering and drug delivery. The presence of HA improves the mechanical and mucoadhesive properties and permits a sustained release of drugs [3-8]. These studies are carried out mostly in the range of concentration where the Pluronic micelles form a gel i.e. at concentrations above 15 vol\%, in the hexagonal phase. A detailed structural characterization of these complex systems is often lacking but dynamic light scattering characterization shows the formation of structures of few hundreds of nanometers [6,9], i.e. more than ten times the micellar size. Before any therapeutic application, the knowledge of the structure appears mandatory, especially if aggregation occurs and might be an issue for injection and circulation into the blood vessels.

From a fundamental point of view, the phase behavior of small colloids and large polymers has attracted much interest in the past years due to its importance in many domains (from nanocomposites to care products) and more specifically for applications in protein crystallization giving to such polymer-colloid systems the name of "protein limit". The counterpart - large colloids and short polymers- is called "colloidal limit". In the latter case, the natural parameter to introduce is the $Q$ parameter defined as $R_{G} / R$, the ratio between the radius of gyration of the polymer and the radius of the colloid $[10,11]$. The colloidal limit is simpler and can be understood by the fact that the polymers are excluded from the space between two adjacent colloids, and exert an unbalanced osmotic pressure which induces an attraction between the particles. In other words, the depletion of the polymers around particles causes entropy loss of polymer when the particles are homogeneously dispersed. The aggregation of the particles increases the accessible volume for the polymers and leads to an entropy gain. In this case, depletion interaction are pairwise additive. The radius of gyration fixes the range of depletion and the polymer concentration sets the strength. The model first proposed by AsakuraOosawa $[10,11]$ and further developed by Vrij [12] gives accurate results for polymer in $\theta$ solvent and $Q<1$. In the protein limit and in a semi-dilute polymer solution, the correlations between the segments of the polymers induce correlations between the positions of the particles which behave as if they 
were attracted to each other [13]. The theoretical treatment and the phase diagram forecast are more difficult since many-body colloidal interactions are involved and there is in addition the necessity to take into account the polymer-polymer interactions and the chain conformation. The first scaling theories were developed the by Sear et al. [14-16]. The main hypotheses are the following: 1- the polymer is considered to be in good solvent conditions and does not adsorb on the particles; 2- all the interactions considered are excluded volume interactions, i.e two polymer segments or two particles or a polymer segment and a particle cannot occupy the same volume. The demixing occurs if the entropy of mixing decreases due to a loss of entropy of translation for the particles or a loss of entropy of conformation for the polymer. For a dilute polymer solution, the chains do not overlap and the system is miscible. When the polymer concentration reaches $c^{*}$ (semi-dilute regime), the chains start to overlap and form a network with a characteristic mesh size or correlation length $\xi[13]$ in which the particles can enter. In consequence, in the protein limit and for polymer in semidilute regime, the particle-polymer interaction depends on the parameter $Q$ defined here as the ratio $D / \xi$ (where $D$ is the particle diameter) and scales with the polymer volume fraction. As the concentration of polymer increases, $\xi$ decreases and the space available to the particle decreases until $\xi \sim D$. In that limit, the free energy to insert a particle and push away the polymer becomes large and drives the phase separation [16]. For $D<\xi$ and low particle concentration, the position of the particles in the polymer solution are correlated over the polymer correlation length as if they were subject to an additional attractive potential. When the particle concentration increases, the correlation between the polymer segments are in turn modified by the particle inducing a drop of the correlation length [17-19]. Further theoretical developments were done within the PRISM (Polymer Reference Interaction Site Model) theory $[20,21]$, which allowed direct determination of thermodynamic and structural properties of the colloid / polymer mixtures. More recently, full-monomer simulations were able to describe the phase boundary for $Q \geq 1$ [22]. Based on the free-volume theory of Lekkerkerker [23] and Bolhuis [22], Fleer and Tuinier [24,25] have derived a general law that allows to predict the binodals and generate a universal scaling law, regardless the polymer and the colloid size.

Most of the experimental studies on depletion concerns nonionic polymers with charged colloids but few is known about the structure and interaction of polyelectrolytes with nonionic colloids. For potential applications as drug delivery systems, it is also mandatory to control the aggregation state and the stability, two factors that may affect the therapeutic efficiency. With this perspective, we are interested in the mixed system composed of Pluronic F127 micelles and HA. Since F127 and HA do not exhibit specific adsorption nor electrostatic interaction, a depletion phenomenon should be observed depending on the polyelectrolyte concentration or the ionic strength that screens the inter- and intrachain electrostatic repulsions. Two HA, with molecular masses of 12 and $300 \mathrm{kDa}$ are used. We work in the Pluronic micellar phase ( 3 vol\%) and different HA volume fractions above $1 \%$, in the semi-dilute regime. The effects of the nature of the salt and its concentration on the formation, shape and organization of the micelles are probed by differential scanning calorimetry (DSC) and small angle neutron scattering (SANS).

\section{Experimental}

\section{Material}

HA (sodium hyaluronate) with two molecular weights, HA12 (12.6 kDa) and HA300 (300 kDa) were kindly provided by Soliance. Pluronic $\mathrm{F} 127, \mathrm{P} 123, \mathrm{NaCl}, \mathrm{NaBr}, \mathrm{KCl}, \mathrm{KBr}, \mathrm{CaCl}_{2}$ and $\mathrm{D}_{2} \mathrm{O}$ were purchased from Sigma-Aldrich and used without further purification. The physical parameters are recalled in Table 1 and 2. 
Table 1: Selected physical parameters of the different chemicals used: chemical formula, molecular weight, density, molecular volume, neutron scattering length density $\rho$. The PO and EO density values are taken as determined in [26].

\begin{tabular}{llcccc}
\hline \multicolumn{1}{c}{ Formula } & $\begin{array}{c}\mathrm{Mw} \\
(\mathrm{g} / \mathrm{mol})\end{array}$ & Density & $\begin{array}{c}\text { Molecular } \\
\text { volume } \\
\left(\AA^{3}\right)\end{array}$ & $\begin{array}{c}\rho \text { (neutron) } \\
\left(\mathrm{cm}^{-2}\right)\end{array}$ \\
\hline F127 & $(\mathrm{EO}) 100(\mathrm{PO}) 65(\mathrm{EO}) 100$ & 12570 & 1.14 & 19000 & - \\
$\mathrm{P} 123$ & $(\mathrm{EO}) 2 \mathrm{PO}(\mathrm{PO}) 65(\mathrm{EO}) 20$ & 5800 & 1.018 & 8700 & - \\
(PO) & $\left(\mathrm{OCH}\left(\mathrm{CH}_{3}\right) \mathrm{CH}_{2}\right)$ & 58 & 1.01 & 96 & $0.3410^{10}$ \\
(EO) & $\left(\mathrm{C}_{2} \mathrm{H}_{4} \mathrm{O}\right)$ & 44 & 1.14 & 62 & $0.6710^{10}$ \\
HA (repeat unit) & $\mathrm{C}_{28} \mathrm{H}_{43} \mathrm{~N}_{2} \mathrm{O}_{23} \mathrm{Na}$ & 401 & 1.695 & 770 & $2.3110^{10}$ \\
solvent & $\mathrm{D}_{2} \mathrm{O}$ & 20 & 1.104 & 30.1 & $6.3610^{10}$
\end{tabular}

Table 2: Characteristics of HA: molecular weight Mw, degree of polymerization DP, polydispersity index PDI, contour length of the fully extended chain Lc calculated with a monomer size of $10.2 \AA \mathrm{f}[27]$.

\begin{tabular}{lcccc}
\hline Name & $\begin{array}{c}\mathrm{Mw} \\
(\mathrm{kDa})\end{array}$ & $\mathrm{DP}$ & $\mathrm{PDI}$ & $\mathrm{L}_{c}(\mathrm{~nm})$ \\
\hline HA12 & 12.6 & 31 & 1.6 & 32 \\
HA300 & 300 & 750 & 2.0 & 760
\end{tabular}

\section{Sample preparation}

The samples are all prepared in $\mathrm{D}_{2} \mathrm{O}$ is order to increase the scattering length density (SLD) contrast and to reduce the incoherent background for the SANS experiments. The sample composition is given by the volume fraction of F127 $\left(\Phi_{\mathrm{F} 127}\right)$ and the volume fraction of $\mathrm{HA}\left(\Phi_{\mathrm{HA}}\right)$ defined as:

$$
\Phi_{F 127}=\frac{V_{F 127}}{V_{F 127}+V_{D 2 O}+V_{H A}} \text { and } \Phi_{H A}=\frac{V_{H A}}{V_{F 127}+V_{D 2 O}+V_{H A}}
$$

Where $\mathrm{V}_{\mathrm{F} 127}, \mathrm{~V}_{\mathrm{D} 2 \mathrm{O}}$ and $\mathrm{V}_{\mathrm{HA}}$ are the volumes of Pluronic, solvent $\left(\mathrm{D}_{2} \mathrm{O}\right.$ or $\mathrm{D}_{2} \mathrm{O}+$ salt $)$ and $\mathrm{HA}$ respectively. In the following, $\Phi_{F 127}$ is kept constant at $3 \%\left(2.610^{-3} \mathrm{~mol} / \mathrm{L}\right)$.

A $3 \% \mathrm{~F} 127$ stock solution in $\mathrm{D}_{2} \mathrm{O}$ or $\mathrm{D}_{2} \mathrm{O}$ brine at the wanted salt concentration is prepared and gently stirred until the total dissolution of F127. The F127 stock solution is then added to the precisely weighted amount of HA to get the final desired concentration. The sample is regularly vortexed and left for equilibration at room temperature 2 days before use. The samples prepared with HA12 flow; with HA300 for concentrations above 2 vol\%, the samples are sufficiently viscous so that they do not flow in an inverted test-tube. At room temperature, close to the F127 critical micellar temperature, all the samples are transparent.

\section{Differential Scanning Calorimetry}

DSC measurements were carried out with a Micro DSC III apparatus (Setaram, France) which allows high sensitivity with dilute solutions. The sample of about $0.4 \mathrm{~g}$ is filled in the Hastelloy vessel and the same amount of $\mathrm{D}_{2} \mathrm{O}$ is used as reference. A first fast heating / cooling cycle at $2 \mathrm{~K} / \mathrm{min}$ between 4 and $60{ }^{\circ} \mathrm{C}$ is performed to ensure the homogeneity of the solution. Then 2 cycles at $0.5 \mathrm{~K} / \mathrm{min}$ are repeated to check for the reproducibility and reversibility of the transition. The critical micellar temperature is 
determined from the temperature at the intersection of the tangent at the first inflection point of the peak with the baseline [28]. The enthalpy of micellization $\left(\Delta \mathrm{H}_{m}\right)$ is calculated from the area of the micellization peak.

\section{Small Angle Neutron Scattering}

Most of the SANS measurements were carried out on the D33 instrument at the Institut Laue Langevin, Grenoble, France. Two wavelengths 6 and $13 \AA$ with a wavelength spread $\Delta \lambda / \lambda$ of $10 \%$ and two detector distances ( $d=2$ and $12 \mathrm{~m}$ ) were used to cover a q-range from $1.510^{-3}$ to $0.45 \AA^{-1}$ where $q$ is the wave vector transfer $(q=4 \pi / \lambda \sin \theta)$ with $2 \theta$ the scattering angle. Additional measurements were carried out on the PAXY instrument at the Laboratoire Léon Brillouin, Saclay, France. Four instrument configurations were used to cover a similar q-range $(\lambda=5 \AA, d=1$ and $3.5 \mathrm{~m} ; \lambda=8 \AA, d=5 \mathrm{~m} ; \lambda=15$ $\AA, d=7 \mathrm{~m}$ ). Samples were placed in $1 \mathrm{~mm}$ thick Hellma cells and thermostatted through a circulation water bath system at $37^{\circ} \mathrm{C}$. Data were corrected from electronic background and empty cell and normalized to the absolute scale $\left(\mathrm{cm}^{-1}\right)$ using standard procedures implemented in the Lamp software at the ILL [29] and in PASINET at the LLB.

\section{SANS data analysis}

In this ternary system as it will be shown in the experimental part, the scattering is fully dominated by the scattering of the micelles for $q<0.15 \AA^{-1}$. Following the approach already used in [30], the general equation used to analyze the scattering intensity of the F127 micelles can be written:

$$
I(q)=\Phi_{m} V P(q) S(q)+I_{\text {coils }}(q)+I_{b c k} \quad \text { Eq. } 1
$$

with $\Phi_{m}$, the volume fraction of particle and $V$ the particle volume, $l_{b c k}$ the incoherent scattering contribution. The form factor $P(q)$ describes the shape of the micelles and $S(q)$ is the interparticle structure factor. $I_{\text {coils }}(q)$ is the scattering of a Gaussian coil. This contribution is added to better taking into account the PEO chains within the micellar shell and the HA macromolecules.

The scattering of the pure Pluronic micelles is fitted to a core-shell model combined with a hard-sphere structure factor. The core contains PPO and water, and the shell PEO and water. The resulting parameters are $R_{c}$, the core radius, $e_{\text {shell, }}$ the thickness of the PEO shell, the core and shell SLD, $\rho_{c}, \rho_{s h}$ and $\Phi_{m}$, the hard sphere volume fraction of micelles. A Gaussian coil with $R_{G}=6 \AA$ is added to improve the fitting at high q $\left(>0.2 \AA^{-1}\right)$, which mainly originates from the PEO segments. Polydispersity (with a Gaussian size distribution) is applied to the core and the shell independently. Finally, the instrument resolution is taken into account in the fitting procedure. The detailed equations of the form and structure factors used for the SANS data analysis are given in the Electronic Supporting Information.

In presence of HA the modification of the scattering intensity at low q values can be explained by a reorganisation of the micelle dispersion state. It is modelled with an attractive square-well structure factor, based on the Baxter's sticky hard-sphere model [31] for the micellar interactions. The potential $V(r)$ has a fine width $\Delta$ and a depth $U_{0}$ (Figure SI 1 ). Considering $r$ the distance between the particles, 
the potential is infinite for $r<\sigma=2 R$; is equal to $-U_{0}$ for $\sigma<r<\sigma+\Delta$ and 0 for $r>\sigma+\Delta$. $R$ is the radius of the micelles; $\sigma=2 \mathrm{R}$ is the diameter of the micelles and $\Delta$ is the range of attraction of the order of the mesh size $\xi$ of the polyelectrolyte network. This model has been successfully used to model the static structure of short-range attractive colloidal systems in a wide range of concentration and temperature [32,33]. At high q values a direct contribution of the HA macromolecule is also observed and taken into account in the Gaussian coil scattering.

\section{Experimental Results}

\section{DSC Characterization}

Typical DSC thermograms for a $3 \% \mathrm{~F} 127$ solution in $\mathrm{D}_{2} \mathrm{O}$ and in $\mathrm{D}_{2} \mathrm{O} / 0.1 \mathrm{M} \mathrm{NaCl}$ during the heating phase are presented in Figure $\mathrm{SI}$ 2. The pronounced endothermic peak is the signature of the micellar formation induced by the dehydration of the PPO as the temperature increases and overcomes the critical micellar temperature. For the reference solution in $\mathrm{D}_{2} \mathrm{O}$, the critical temperature is found at $22^{\circ} \mathrm{C}$ in good agreement with values reported in the literature [34,35]. Figure 1 shows the effect of the salt concentration $(\mathrm{NaCl})$ on the micellization temperatures without and with $\mathrm{HA}\left(\Phi_{\mathrm{HA}}=2 \%\right)$. Figure 2 shows the effect of $\mathrm{HA}$ concentration at a fixed $\mathrm{NaCl}$ concentration of $0.1 \mathrm{M}$. In Figure $\mathrm{SI} 3$, the nature of the salt is investigated. It is well established that salt affects the water solvency. Water structuring salts, leading to an increase in self hydration through hydrogen bounding reduce the hydration of PPO and decrease its water solubility resulting in a lower critical micellar temperature. $\mathrm{NaCl}$ and $\mathrm{KCl}$ have a slight larger effect than $\mathrm{NaBr}$ and $\mathrm{KBr}$. A linear relationship between the critical temperature and the salt concentration is observed as already reported [36,37]. Independently of its molecular mass, addition of the HA (polyanions and counterions) affects the transition in the same manner as a low molecular weight electrolyte, inducing a linear decrease of the critical temperature as its concentration increases. For all the samples, the enthalpy of micellization remains constant within the experimental errors with an average value of $\Delta \mathrm{H}_{\mathrm{m}}=355 \mathrm{~kJ} / \mathrm{mol}$. In the following of the paper, the experiments are performed at $37^{\circ} \mathrm{C}$ in a domain well above the critical micellar temperature for all the salt and $\mathrm{HA}$ concentrations investigated.

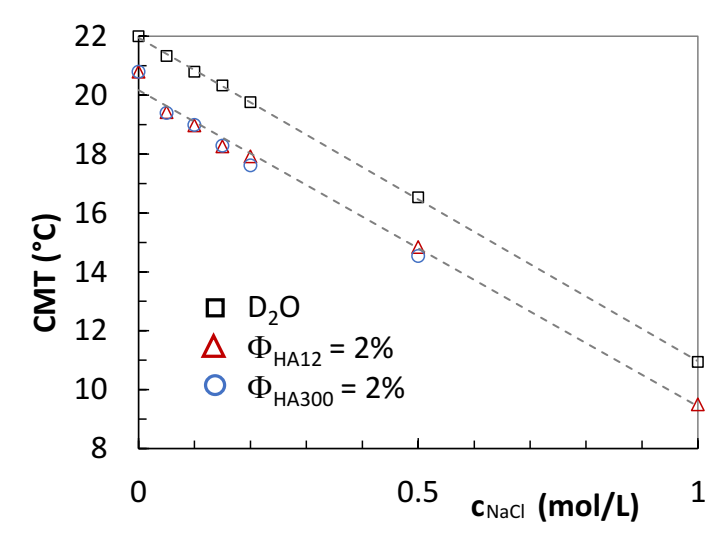

Figure 1: Evolution of the F127 critical micellar temperature (determined by DSC) as a function of the $\mathrm{NaCl}$ concentration $\left(\mathrm{c}_{\mathrm{NaCl}}\right)$. $(\square$ black $) \Phi_{\mathrm{F} 127}=3 \%$; $(\triangle$ red $) \Phi_{\mathrm{F} 127}=3 \%, \Phi_{\mathrm{HA} 12}=2 \%$; (O blue $) \Phi_{\mathrm{F} 127}=3 \%$, $\Phi_{\text {HA300 }}=2 \%$. The dotted lines are guides for the eyes. 


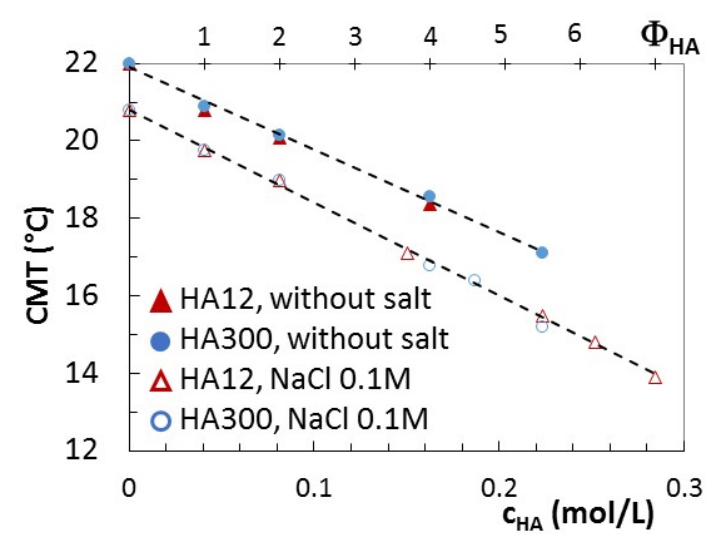

Figure 2: Evolution of the F127 critical micellar temperature (determined by DSC) as a function of the $\mathrm{HA}$ concentration $\left(\mathrm{C}_{\mathrm{HA}}\right)$ or volume fraction $\left(\Phi_{\mathrm{HA}}\right)$. $(\Delta$ red $) \Phi_{\mathrm{F} 127}=3 \%$ and $\mathrm{HA} 12$ in $\mathrm{D}_{2} \mathrm{O} ;(\bullet$ blue $) \Phi_{\mathrm{F} 127}=$ $3 \%$ and $\mathrm{HA300}$ in $\mathrm{D}_{2} \mathrm{O} ;\left(\triangle\right.$ red) $\Phi_{\mathrm{F} 127}=3 \%$ and $\mathrm{HA} 12$ in $\mathrm{D}_{2} \mathrm{O} / 0.1 \mathrm{M} \mathrm{NaCl} ;\left(\mathrm{O}\right.$ blue) $\Phi_{\mathrm{F} 127}=3 \%$ and $\mathrm{HA} 300$ in $\mathrm{D}_{2} \mathrm{O} / 0.1 \mathrm{M} \mathrm{NaCl}$.

\section{SANS Structural Characterization}

\section{Characterization of the individual components}

In this paragraph, we briefly present the characterization of the binary systems (i.e., pure Pluronic or HA solutions) before to move to the ternary system.

\section{Pluronic F127}

The scattering of the $3 \% \mathrm{~F} 127$ solution in $\mathrm{D}_{2} \mathrm{O}$ is shown in Figure $\mathrm{SI} 4$ together with the data analysis. At high angles, the nearly $q^{-2}$ behavior comes from the Gaussian behavior of the PEO chains in the hydrophilic shell. A correlation peak is clearly visible at $q=0.018 \AA^{-1}$, corresponding in the real space to a distance of $350 \AA$ \& between the micelles. The fit to the core-shell form factor described above, gives a core radius of $45 \AA$ and a shell thickness of $60 \AA$. The experimental shell SLD found at $5.810^{10} \mathrm{~cm}^{-2}$ corresponds to $87 \%$ of hydration: the shell is almost invisible by SANS. Due to the high hydration level, the micellar volume fraction $\Phi_{m}$ within the core-shell description is equal to $11 \%$, a value significantly higher than the volume of the dry Pluronic. The core SLD is equal to $0.810^{10} \mathrm{~cm}^{-2}$, corresponding to $10 \%$ of water. These values are in good agreement with previous studies on Pluronics $[30,38,39]$.

The effect of salt ( $\mathrm{NaCl}$ ) for concentrations up to $4 \mathrm{M}$ is presented in Figure $\mathrm{SI} 5$. Up to $0.5 \mathrm{M} \mathrm{NaCl}$, the curves are all superimposed, the size, shape and organization of the micelles are not modified. The SLD of the shell remain at $5.810^{10} \mathrm{~cm}^{-2}$ but that of the core decreases to $0.410^{10} \mathrm{~cm}^{-2}$, close to that of the PPO. Within the accuracy of the measurements and data analysis, the core does not contain water anymore. Between 1 and $2 \mathrm{M}$, one observes the shift of the oscillation toward lower q together with the increase of the intensity at small $\mathrm{q}$ which indicates a growth of the micelles. From 2 to $4 \mathrm{M} \mathrm{a}$ transition from spherical to cylindrical micelles occurs induced by the dehydration of the PEO segments in agreement with the literature $[40,41]$.

Finally, Figure $\mathrm{SI} 6$ shows that the 4 monovalent salts investigated $(\mathrm{NaCl}, \mathrm{NaBr}, \mathrm{KCl}$ and $\mathrm{KBr}$ ) at $0.1 \mathrm{M}$ as well as the divalent salt $\mathrm{CaCl}_{2}$ at $0.03,0.05$ and $0.1 \mathrm{M}$ do not modify the scattering of the micelles. 


\section{Hyaluronic Acid}

$H A$ is a semirigid polyelectrolyte. The total persistence length $L_{T}$ is the sum of two contributions, the intrinsic persistence length, $L_{0}$ and the electrostatic persistence length $L_{e}$. $L_{e}$ depends on the ionic strengthfixed by the dissociated counterions and the added salt concentrations. It can be calculated with the model developed by Odjik, Skolnick and Fixman [42-44]. Assuming a fully stretched polyion (rod limit) with a contour length $\mathrm{L}_{c}$, the radius of gyration is equal to $R_{G}=L_{c} / \sqrt{12}$ and the overlap concentration is given by $\mathrm{c}^{*}=M \mathrm{w} /\left[\frac{4}{3} \pi R_{G}^{3} N_{a}\right]$. One obtains $\mathrm{c}^{*}$ values of 6 and $1.210^{-2} \mathrm{~g} / \mathrm{L}$ for HA12 and HA300 respectively. In a more realistic case, by considering a "wormlike" chain of persistence length $\mathrm{L}_{\mathrm{T}}$ (with $\mathrm{L}_{\mathrm{T}}=90 \AA$ ), the Benoit and Doty equation $[45,46]$ gives $R_{G}$ of $69 \AA$ and $470 \AA$ leading to $\mathrm{c}^{*}$ values of 15 and $1.1 \mathrm{~g} / \mathrm{L}$ for HA12 and HA300. These values are only indicative. However in the following, at HA concentrations higher than $1 \mathrm{vol} \%(16.9 \mathrm{~g} / \mathrm{L})$ we can admit that we are always above $\mathrm{c}^{*}$.

In the case of a depletion mechanism as recalled in the introduction, the mesh size of the polymer network $\xi$ is used to quantify the width of the attractive potential. In order to estimate thes correlation length, scattering experiments have been carried out on $\mathrm{HA} 12$ and $\mathrm{HA} 300$ solutions (2\%) in $\mathrm{D}_{2} \mathrm{O}$ and in $0.1 \mathrm{M} \mathrm{NaCl}$. The results are presented in Figure SI 7 and SI 8. Below $0.01 \AA^{-1}$ one observes an upturn whose origin has been largely debated, and is related to the formation of large aggregates $[27,46,47]$. In absence of salt and more clearly for the SAXS data, the so-called polyelectrolyte peak is visible. Its position $\mathrm{q}_{\mathrm{m}}$ provides a direct measurement of the correlation length $\xi\left(\xi=2 \pi / \mathrm{q}_{\mathrm{m}}\right)$. It is close to $80 \AA$ independently of the molecular mass of the polyelectrolyte as we are in the semi-dilute regime.

In presence of $0.1 \mathrm{M}$ of salt, the peak disappears due to the screening of the electrostatic repulsions between the chain segments [48]. HA chains adopt a random coil configuration with a persistence length close to the intrinsic one [27]. This persistence length has been estimated by light scattering and SANS in dilute conditions (below the overlap concentration $\left.c^{*}\right)$ and at high ionic strength $(0.1 \mathrm{M}$ $\mathrm{NaCl}$ ) when all the electrostatic contributions are screened so that the electrostatic persistence length $L_{e}$ is negligible and the total persistence length $L_{T}$ is equal to the intrinsic persistence length $L_{0}$. The different studies report $L_{0}$ values between 50 and $90 \AA[49,50]$. This remains comparable to the radius of the F127 micelles and for this intrinsic rigidity we can infer that the chain cannot wrap around a single micelle. For semi-dilute solution of neutral polymer, the scattering length function can be described by the Ornstein-Zernike equation leading to the extraction of the correlation length $\xi$. We admit that this behavior is still valid for $\mathrm{HA}$ in presence of $0.1 \mathrm{M} \mathrm{NaCl}$. In good solvent conditions, $\xi$ scales with the concentration $c$ according [46]:

$$
\xi \sim c^{-0.77}
$$

Eq. 2

It should be noticed that for hyaluronan, a larger exponent of 0.9 has been measured in [27] and it is explained by the fact that the behavior of the chains is close to Gaussian chains without excluded volume effects.

The weakness of the signal in the SANS data with the upturn at low $q$, does not allow any accurate determination of the correlation length. In the following, it will be estimated at $\xi_{0} \approx 60 \AA$ from the SAXS data for the $2 \% \mathrm{HA}$ concentration, a value slightly smaller than the correlation length in absence of salt. The mesh size for a HA concentration $c$ (in vol\%) is therefore obtained following:

$$
\xi=\xi_{0}(c / 2)^{-0.77}
$$

The mesh size value is taken as an estimation of the width $\Delta$ of the square-well potential. 


\section{Characterization of the ternary system}

\section{Effect of salt, HA concentration and molar mass}

SANS data of $\mathrm{F} 127$ at $\Phi_{\mathrm{F} 127}=3 \%$ in pure $\mathrm{D}_{2} \mathrm{O}$ as a function of the volume fraction of $\mathrm{HA}$ are shown in Figure 3 (left) for HA12 and Figure 4 (left) for HA300. The signal is fully dominated by the scattering of the micelles and is almost not modified by the presence of HA. We observe a small increase of the intensity at low q for $\Phi_{\text {HA300 }}>4 \%$. Also, at high q above $0.15 \AA^{-1}$, slight modifications are observed due to the scattering of polyelectrolyte. In the middle q domain, the overlap between the curves is the evidence that the size and the shape of the micelles are not modified by the presence of HA.

The situation considerably differs in presence of $0.1 \mathrm{M}$ of $\mathrm{NaCl}$ (figure 3 and 4 , right). As in pure $\mathrm{D}_{2} \mathrm{O}$, the small increase of the intensity at high $q$ is the signature of the HA chains. In the middle $q$ range, the scattering of the micelles dominates and all the curves are superimposed. The oscillation of the form factor remains at the same q-position. At low q, one observes a significant increase of the scattering intensity that reaches a plateau. This is the signature of small clusters of micelles in the solution. The effect of HA differs thus from a salt effect (Figure SI 4) inducing the transition from spherical to cylindrical micelles. Above volume fractions of $\Phi_{\mathrm{HA}}=9 \%$ and $5 \%$ for $\mathrm{HA} 12$ and HA300 respectively, the samples become turbid and the scattering curves present the characteristic diffraction peaks of a face centered cubic phase. It should be noted that due to the viscosity of the samples, there is no macroscopic phase separation. From the first peak position (corresponding to the 111 reflection), we calculate a center-to-center distance between the micelles of $213 \AA$ and $208 \AA$ for $\mathrm{HA} 300$ at 5 and $6 \%$ respectively and $198 \AA$ with HA12 at $9 \%$. The micelles are closely packed. This crystalline structure is usually obtained in pure F127 in salt free water solutions at volume fractions higher than $20 \%$ [51].

Below the HA concentration leading to the crystallization of micelles, the scattering data are fitted with a core-shell model for the form factor and a square-well structure factor, enabling to reproduce the micelle aggregation and the cluster formation. These different contributions are shown in Figure SI 9. The potential width $\Delta$ is fixed to the mesh size of the HA network according to Eq.3. The results are shown with full lines in Figure 3 and Figure 4 for HA12 and HA300 respectively and in Figure SI 10 with a shift in intensity to improve the visual clarity. The summaries of the fit parameters are given in Table 3 and Table 4. The data analysis confirms that the size of the micelles is not modified by the presence of HA. From the position of the maximum of the structure factor, an average distance between the center of mass of the micelles in the cluster is calculated $\left(D_{m}\right)$. It decreases from $350 \AA$ without $H A$ to $168 \AA$ and $198 \AA$ at the highest HA12 and HA300 concentrations just before their crystallization. The PEO shells are slightly overlapping and this explain the small decrease down to $5.610^{-6} \AA^{-2}$ of the average SLD. Finally, the increase of the scattering at low $q$ is consistent with an increase of the depth of the potential and therefore to more attractive interactions between the micelles. At a given $\mathrm{HA}$ volume fraction, the potential is deeper for $\mathrm{HA} 300$ than HA12, and the crystallization of the micelles occurs at lower volume fraction for HA300 ( 5\%) than for HA12 ( 9\%). The number of micelles per clusters $\mathrm{N}_{\text {mic }}$ is estimated from the radius of gyration of the clusters calculated from the Guinier regime at low q (Eq SI 8). It varies between 1 and 25 as the HA concentration increases. For $\Phi_{\text {HA12 }} \leq 2 \%, N_{\text {mic }}$ is found at 1 with however a distance between adjacent micelles of $225 \AA$ (against $350 \AA$ in absence of $\mathrm{HA})$. This shows that the micelles are no longer homogeneously distributed in the solution. This number is also an average, and it cannot be excluded that single micelles and clusters are already coexisting. The size of the micellar clusters grows when the HA concentration increases. At a fixed HA concentration, the size of the clusters is larger with the largest polyelectrolyte molecular mass. 
A complementary measurement shows that by increasing the Pluronic concentration to $5 \%$, for a same HA300 volume fraction, the attractive interaction between the micelles is larger and the micellar cristallisation occurs at a smaller volume fraction of HA (between 2 and 4\%) (Figure SI 11 and table SI 1).
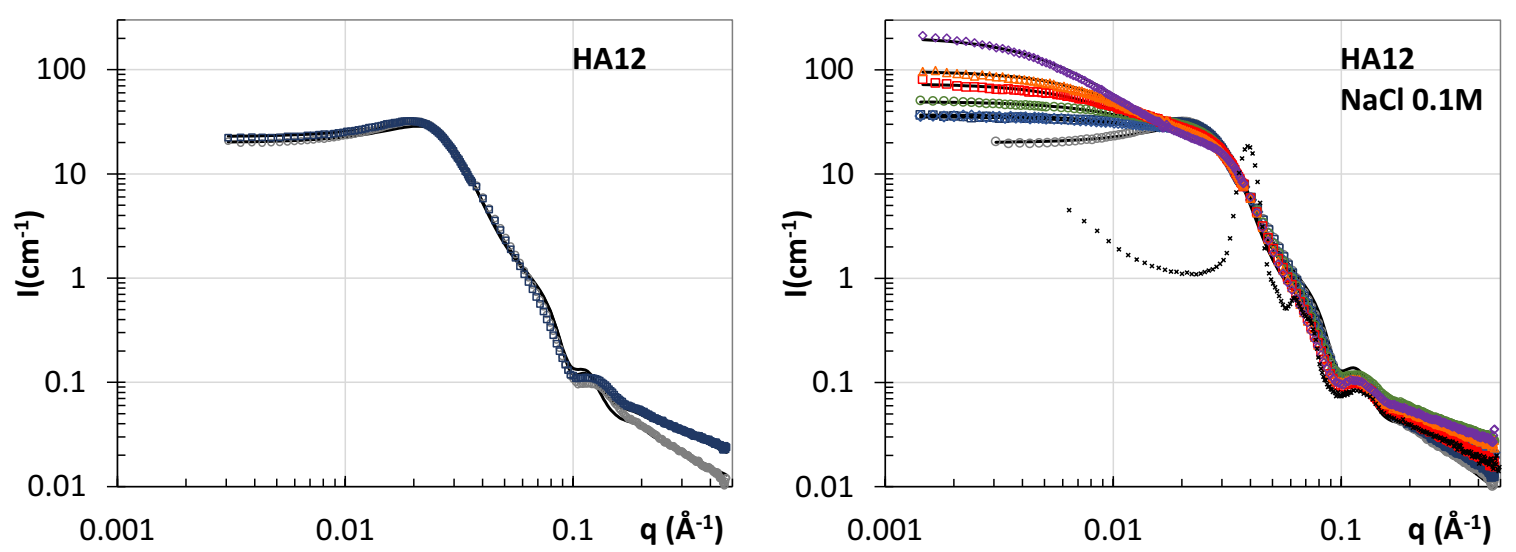

Figure 3: Evolution of the scattered intensity as a function of the volume fraction of HA12. $\Phi_{\mathrm{F} 127}=3 \%$. $\mathrm{T}=37^{\circ} \mathrm{C}$. Left: in $\mathrm{D}_{2} \mathrm{O}$. ( $\mathrm{O}$ grey) $\Phi_{\mathrm{HA}}=0 \%$, ( $\square$ dark blue) $\Phi_{\mathrm{HA}}=2 \%$. Right: in $\mathrm{NaCl} 0.1 \mathrm{M}$. ( $\mathrm{O}$ grey) $\Phi_{\mathrm{HA}}$ $=0 \%,(\square$ dark blue $) \Phi_{\mathrm{HA}}=1 \%,(\diamond$ blue $) \Phi_{\mathrm{HA}}=2 \%,(\bigcirc$ green $) \Phi_{\mathrm{HA}}=4 \%,(\square$ red $) \Phi_{\mathrm{HA}}=5 \%,(\triangle$ orange $)$ $\Phi_{\mathrm{HA}}=6 \%$, ( $\diamond$ purple) $\Phi_{\mathrm{HA}}=7 \%$, (+ black) $\Phi=9 \%$. Full lines correspond to the best fits of the scattering data before crystallization threshold.
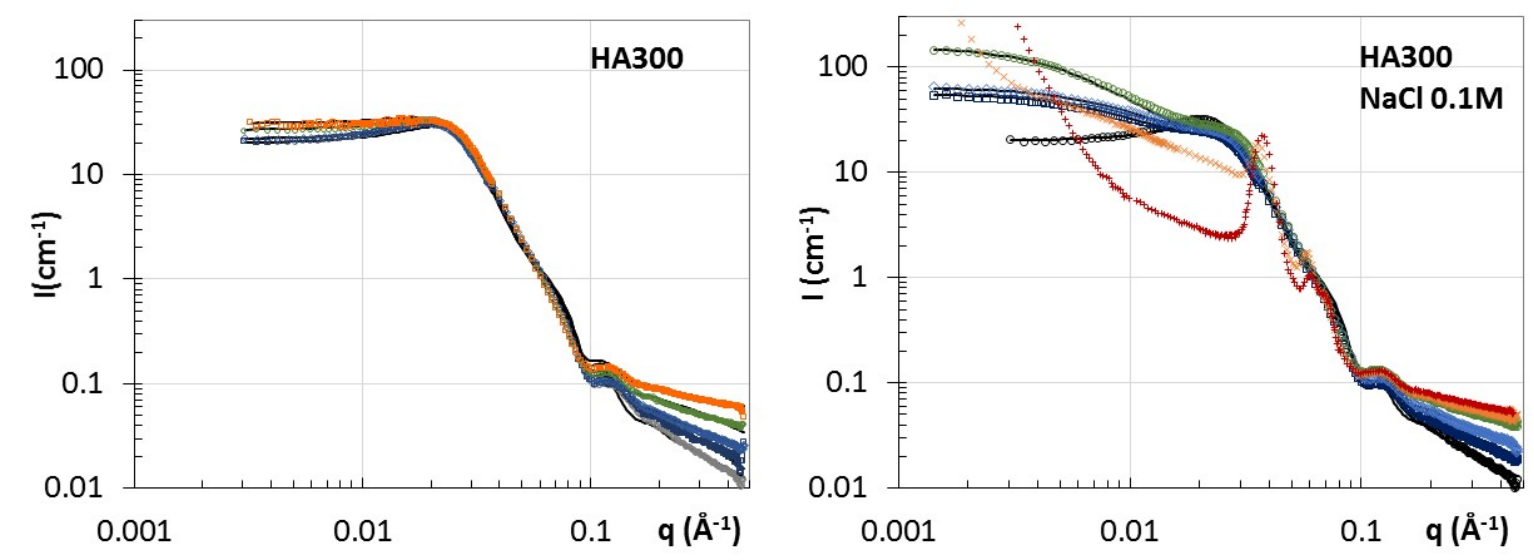

Figure 4: Evolution of the scattered intensity as a function of the volume fraction of HA300. $\Phi_{127}=3 \%$. $\mathrm{T}=37^{\circ} \mathrm{C}$. Left: in $\mathrm{D}_{2} \mathrm{O}$. (O grey) $\Phi_{\mathrm{HA}}=0 \%,(\square$ dark blue $) \Phi_{\mathrm{HA}}=1 \%$, $(\diamond$ blue $) \Phi_{\mathrm{HA}}=2 \%,(\bigcirc$ green $) \Phi_{\mathrm{HA}}$ $=4 \%$, $(\square$ orange $) \Phi_{\mathrm{HA}}=5.5 \%$. Right: in $\mathrm{NaCl} 0.1 \mathrm{M}$. (O black) $\Phi_{\mathrm{HA}}=0 \%$, $(\square$ dark blue $) \Phi_{\mathrm{HA}}=1 \%$, $(\diamond$ blue) $\Phi_{\mathrm{HA}}=2 \%$, (O green) $\Phi_{\mathrm{HA}}=4 \%$, (+ orange) $\Phi_{\mathrm{HA}}=5 \%$, ( $\square$ red ) $\Phi_{\mathrm{HA}}=6 \%$. Full lines correspond to the best fits of the scattering data before crystallization threshold. 
Table 3: $\mathrm{F} 127 \Phi_{\mathrm{F} 127}=3 \%, \mathrm{NaCl} 0.1 \mathrm{M}, \mathrm{HA} 12, \mathrm{~T}=37^{\circ} \mathrm{C}$. Effect of $\mathrm{HA}$ concentration. Fit parameters from the SANS data analysis to a core-shell model for the form factor and a square-well potential for the structure factor. Distance between micelles calculated from the position of the first maximum of the structure factor. $\Phi_{\mathrm{HA}}$ : $\mathrm{HA}$ volume fraction; $\Phi_{m}$ : volume fraction of micelles; Rc: core radius; $e_{s h}$ : shell thickness; $\rho_{c}, \rho_{s h}, \rho_{\text {solv }}$ : scattering length densities of the core, shell and solvent; $D_{m}$ : average distance between micelles; $N_{m i c}:$ number of micelles in cluster; $\xi$ : square-well potential width; $\mathrm{U}_{0}$ : depth of the square-well potential.

\begin{tabular}{l|ccccccc}
$\boldsymbol{\Phi}_{\text {HA }}$ & $\mathbf{0}$ & $\mathbf{1 \%}$ & $\mathbf{2 \%}$ & $\mathbf{4 \%}$ & $\mathbf{5 \%}$ & $\mathbf{6 \%}$ & $\mathbf{7 \%}$ \\
\hline$\Phi_{\mathrm{m}}$ & 0.11 & 0.11 & 0.1 & 0.1 & 0.092 & 0.085 & 0.09 \\
$\operatorname{Rc}(\AA)$ & 45 & 45 & 45 & 46 & 47 & 47 & 47 \\
$\mathrm{e}_{\text {sh }}(\AA)$ & 60 & 60 & 60 & 60 & 60 & 60 & 60 \\
$\rho_{\mathrm{c}}\left(10^{-6} \AA^{-2}\right)$ & 0.8 & 0.4 & 0.4 & 0.4 & 0.4 & 0.4 & 0.4 \\
$\rho_{\text {sh }}\left(10^{-6} \AA^{-2}\right)$ & 5.8 & 5.8 & 5.8 & 5.8 & 5.8 & 5.6 & 5.6 \\
$\rho_{\text {solv }}\left(10^{-6} \AA^{-2}\right)$ & 6.4 & 6.4 & 6.4 & 6.4 & 6.4 & 6.4 & 6.4 \\
$D_{m}(\AA)$ & 350 & 224 & 215 & 208 & 198 & 178 & 168 \\
$N_{\text {mic }}$ & 1 & 1 & 1 & 2 & 5 & 9 & 25 \\
$\xi(\AA)$ & - & 75 & 60 & 47 & 44 & 41 & 40 \\
$U_{0}\left(k_{B} T\right)$ & - & 0.5 & 0.66 & 1.34 & 2.1 & 2.5 & 3.7
\end{tabular}

Table 4: $\mathrm{F} 127 \Phi_{\mathrm{F} 127}=3 \%, \mathrm{NaCl} 0.1 \mathrm{M}, \mathrm{HA} 300, \mathrm{~T}=37^{\circ} \mathrm{C}$. Effect of HA concentration. Fit parameters from the SANS data analysis to a core-shell model for the form factor and a square-well potential for the structure factor. Same parameter definition as in Table 3.

\begin{tabular}{l|ccc}
$\boldsymbol{\Phi}_{\text {HA }} \%$ & $\mathbf{1 \%}$ & $\mathbf{2 \%}$ & $\mathbf{4 \%}$ \\
\hline$\Phi_{\mathrm{m}}$ & 0.10 & 0.11 & 0.11 \\
$\mathrm{R}_{\mathrm{c}}(\AA)$ & 45 & 45 & 45 \\
$\mathrm{e}_{\text {sh }}(\AA)$ & 60 & 60 & 60 \\
$\rho_{\mathrm{c}}\left(10^{-6} \AA^{-2}\right)$ & 0.4 & 0.4 & 0.4 \\
$\rho_{\text {sh }}\left(10^{-6} \AA^{-2}\right)$ & 5.8 & 5.8 & 5.8 \\
$\rho_{\text {solv }}\left(10^{-6} \AA^{-2}\right)$ & 6.4 & 6.4 & 6.4 \\
$D_{\mathrm{m}}(\AA)$ & 214 & 207 & 198 \\
$\mathrm{~N}_{\text {mic }}$ & 5 & 6 & 20 \\
$\xi(\AA)$ & 75 & 60 & 47 \\
$U_{0}\left(\mathrm{k}_{\mathrm{B}} \mathrm{T}\right)$ & 1 & 1.45 & 2.8
\end{tabular}

\section{Effect of the salt ( $\mathrm{NaCl}$ ) concentration}

In this part, the effect of the salt $(\mathrm{NaCl})$ concentration is explored. The volume fraction of $\mathrm{HA}$ is fixed at $2 \%$ and the salt concentration kept below $1 \mathrm{M}$, to avoid the growth of the micelles and their shape transition (Figure SI 5). The scattering results are presented in Figure 5 and Figure SI 12. The fitting parameters are summarized in Tables 5 and 6 . For HA chains, above salt concentration of $0.1 \mathrm{M}$, the electrostatic persistence length $L_{e}$ is reduced to $1.5 \AA$ [46] and the total chain stiffness is only due to the intrinsic stiffness of the chain. The folding of the HA network is mostly complete [52] and the value of the potential width is kept constant at $60 \AA$. The size of the cluster increases with the salt concentration. At a fixed salt concentration, before the crystallization, the clusters are larger with HA300 than with HA12. Consequently, the attractive potential determined from the data analysis is deeper for HA300. It should be pointed that the $0.5 \mathrm{M}$ concentration with HA12 corresponds certainly 
to a concentration very close to the 2-phases domain as shown with a drastic increase of the forward scattered intensity. Inside the clusters, by increasing the salt concentration from $0.05 \mathrm{M}$ to $0.5 \mathrm{M}$, the center-to-center distance of the micelles decreases from 224 to $200 \AA$. The micelles are then in contact.

The micelle crystallization occurs at lower salt concentration for HA300 (>0.1 M) than HA12 (>0.5 M). For HA12, as the sample remains relatively fluid, a phase separation occurs, in which the supernatant is enriched in micelles almost in contact (Figure SI 13). With HA300, the sample is viscous, there is no macroscopic phase separation. From the first Bragg peak position, considering a face centered cubic lattice, we calculate a center-to-center distance of $235 \AA$ A between the micelles, thus slightly larger than their diameter.
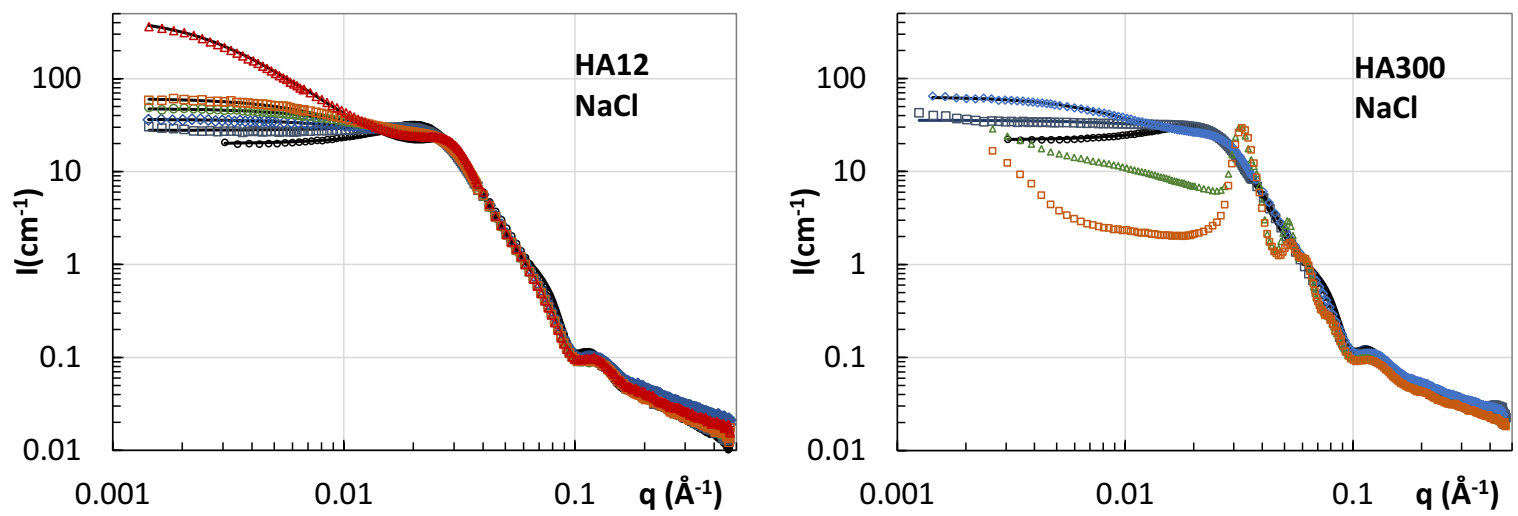

Figure 5: Evolution of the scattered intensity for a solution of $\Phi_{\mathrm{F} 127}=3 \%$, as a function of the $\mathrm{NaCl}$ concentration. $\mathrm{T}=37^{\circ} \mathrm{C}$. Left: $\mathrm{HA} 12 \Phi_{\text {HA12 }}=2 \%$. (O black) $\mathrm{NaCl} 0 \mathrm{M}$, ( $\square$ dark blue) $\mathrm{NaCl} 0.05 \mathrm{M}$, ( $\diamond$ blue $)$ $\mathrm{NaCl} 0.1 \mathrm{M}$, (O green) $\mathrm{NaCl} 0.15 \mathrm{M}$, ( $\square$ orange) $\mathrm{NaCl} 0.2 \mathrm{M}$, $\left(\triangle\right.$ red) $\mathrm{NaCl} 0.5 \mathrm{M}$. Right: $\Phi_{\text {HA300 }}=2 \%$. (black) $\mathrm{NaCl} 0 \mathrm{M}$, ( $\square$ dark blue) $\mathrm{NaCl} 0.05 \mathrm{M}$, ( $\diamond$ blue) $\mathrm{NaCl} 0.1 \mathrm{M}$, (O green) NaCl $0.15 \mathrm{M}$, ( $\square$ orange) $\mathrm{NaCl} 0.2 \mathrm{M}$. Full lines correspond to the best fits of the scattering data before crystallization threshold.

Table 5: $\mathrm{F} 127 \Phi_{\mathrm{F} 127}=3 \%, \mathrm{HA} 12, \Phi_{\mathrm{HA}}=2 \%, \mathrm{~T}=37^{\circ} \mathrm{C}$. Effect of the $\mathrm{NaCl}$ concentration. Fit parameters from analysis the SANS data to a core-shell model for the form factor model and a square-well potential for the structure factor. Same parameter definition as in Table 3.

\begin{tabular}{l|ccccc}
$\mathrm{C}_{\mathrm{Nacl}}(\mathbf{m o l} / \mathrm{L})$ & $\mathbf{0 . 0 5}$ & $\mathbf{0 . 1}$ & $\mathbf{0 . 1 5}$ & $\mathbf{0 . 2 0}$ & $\mathbf{0 . 5 0}$ \\
\hline$\Phi_{\mathrm{m}}$ & 0.1 & 0.1 & 0.1 & 0.1 & 0.1 \\
$\operatorname{Rc}(\AA)$ & 45 & 45 & 45 & 45 & 45 \\
$\mathrm{e}_{\text {sh }}(\AA)$ & 60 & 60 & 60 & 60 & 60 \\
$\rho_{\mathrm{c}}\left(10^{-6} \AA^{-2}\right)$ & 0.4 & 0.4 & 0.4 & 0.4 & 0.4 \\
$\rho_{\text {sh }}\left(10^{-6} \AA^{-2}\right)$ & 5.8 & 5.8 & 5.8 & 5.8 & 5.8 \\
$\rho_{\text {solv }}\left(10^{-6} \AA^{-2}\right)$ & 6.4 & 6.4 & 6.4 & 6.4 & 6.4 \\
$\mathrm{D}_{\mathrm{m}}(\AA)$ & 224 & 217 & 209 & 207 & 201 \\
$\mathrm{~N}_{\text {mic }}$ & 1 & 1 & 3 & 5 & 80 \\
$\xi(\AA ̊)$ & 60 & 60 & 60 & 60 & 60 \\
$\mathrm{U}_{0}\left(\mathrm{k}_{\mathrm{B}} \mathrm{T}\right)$ & 0.038 & 0.66 & 1.05 & 1.4 & 2.33
\end{tabular}


Table 6: $\mathrm{F} 127 \Phi_{\mathrm{F} 127}=3 \%, \mathrm{HA} 300, \Phi_{\mathrm{HA}}=2 \%, \mathrm{~T}=37^{\circ} \mathrm{C}$. Effect of the $\mathrm{NaCl}$ concentration. Fit parameters to a coreshell form factor model and a square-well potential for the structure factor. Same parameter definition as in Table 3.

\begin{tabular}{l|cc}
$\mathbf{C}_{\text {Nacl }}(\mathbf{m o l} / \mathrm{L})$ & $\mathbf{0 . 0 5}$ & $\mathbf{0 . 1}$ \\
\hline$\Phi_{\mathrm{m}}$ & 0.10 & 0.11 \\
$\operatorname{Rc}(\AA)$ & 45 & 45 \\
$\mathrm{e}_{\mathrm{sh}}(\AA)$ & 60 & 60 \\
$\rho_{\mathrm{c}}\left(10^{-6} \AA^{-2}\right)$ & 0.4 & 0.4 \\
$\rho_{\mathrm{sh}}\left(10^{-6} \AA^{-2}\right)$ & 5.8 & 5.8 \\
$\rho_{\text {solv }}\left(10^{-6} \AA^{-2}\right)$ & 6.4 & 6.4 \\
$D_{\mathrm{m}}(\AA)$ & 228 & 208 \\
$\mathrm{~N}_{\text {mic }}$ & 1 & 6 \\
$\xi(\AA)$ & 60 & 60 \\
$U_{0}\left(\mathrm{k}_{\mathrm{B}} T\right)$ & 0.4 & 1.48
\end{tabular}

\section{Effect of the nature of the salt}

Does the nature of salt impact the micellar stability and organization? We work at volume fractions of $3 \%$ for $\mathrm{F} 127$ and $2 \%$ for $\mathrm{HA}$. Four monovalent salts, $\mathrm{NaCl}, \mathrm{KCl}, \mathrm{NaBr}, \mathrm{KBr}$ at $0.1 \mathrm{M}$ are used and the divalent salt $\mathrm{CaCl}_{2}$ is chosen for its relevance in biological systems. For further comparison with the monovalent salts, three concentrations, $0.033,0.05$ and $0.1 \mathrm{M}$ are investigated, corresponding to ionic strengths of $0.1,0.15$ and $0.3 \mathrm{M}$. The SANS data are presented in Figure 6 and 7 and Figure SI 14.

In presence of the monovalent salts the scattering curves are almost identical with HA12. The data analysis show that the attractive potential is slightly higher for $\mathrm{NaBr}$ and $\mathrm{KBr}\left(0.7 \mathrm{k}_{\mathrm{B}} \mathrm{T}\right)$ compared to $\mathrm{NaCl}$ and $\mathrm{KCl}\left(0.6 \mathrm{k}_{\mathrm{B}} \mathrm{T}\right)$. With $\mathrm{HA300}$, the scattering curves are almost superimposed for $\mathrm{NaCl}$ and $\mathrm{KCl}$, and superimposed for $\mathrm{NaBr}$ and $\mathrm{KBr}$ with an attractive potentials equal to 1.2-1.3 $\mathrm{k}_{\mathrm{B}} \mathrm{T}$ for the former salts and $1.8 \mathrm{k}_{\mathrm{B}} \mathrm{T}$ for the latter ones. The clusters are significantly larger with HA300 than with HA12. The center-to-center distance between the micelles is also a few Angströms smaller with $\mathrm{NaBr}$ and $\mathrm{KBr}$ than with $\mathrm{NaCl}$ and $\mathrm{KCl}$. For $\mathrm{HA} 12$ with $\mathrm{NaCl}$ and $\mathrm{KCl}$, the micelles are separated by a tenth of Angströms, in the other cases, the micelles are in contact.

In presence of the divalent salt $\mathrm{CaCl}_{2}$, the ionic strength is the relevant parameter. The 0.1 and $0.15 \mathrm{M}$ concentration with monovalent salts can be compared with the 0.03 and $0.05 \mathrm{M}$ concentrations with $\mathrm{CaCl}_{2}$. For both $\mathrm{HA} 12$ and $\mathrm{HA} 300$, the divalent salt induces a larger attractive potential than the monovalent salts, and thus larger cluster size. In the cluster, the micelles are in contact. For HA300, the crystallization of the micelles occurs between 0.1 and $0.15 \mathrm{M}$ in ionic strength as previously observed with $\mathrm{NaCl}$ (Figure 5). Their center-to-center distances are $243 \AA$ in $0.05 \mathrm{M} \mathrm{CaCl}_{2}$ solution and $236 \AA$ in $0.1 \mathrm{M} \mathrm{CaCl}_{2}$ solution. 

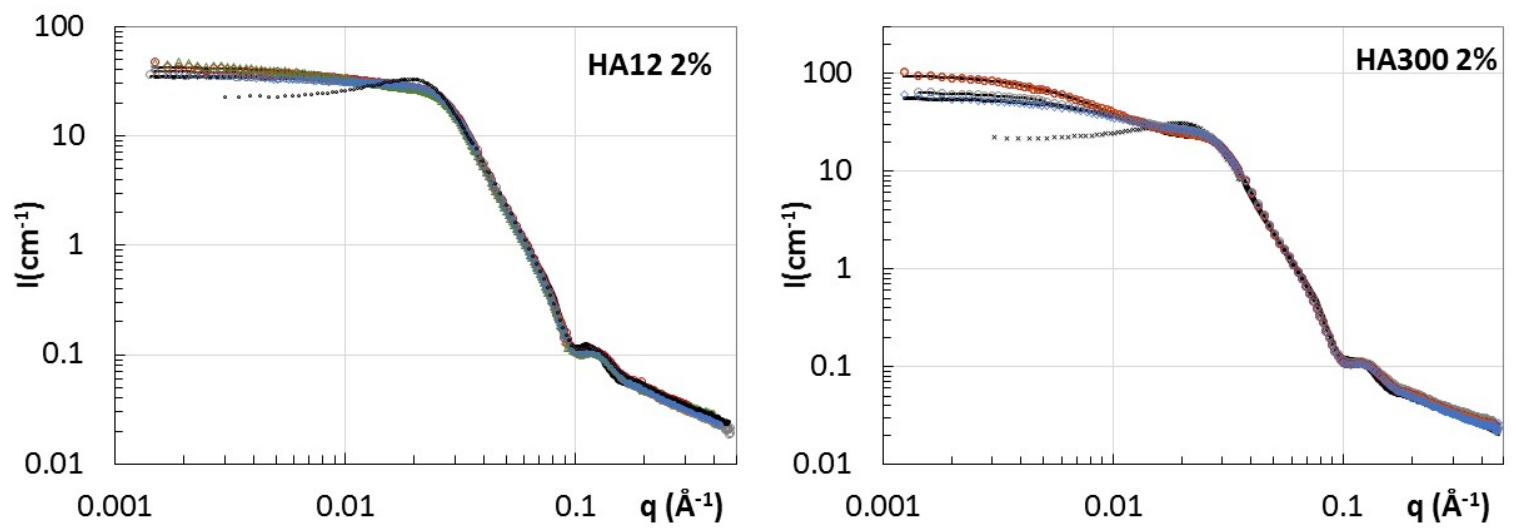

Figure 6: SANS data showing the effect of the nature of the salt. $\Phi_{\mathrm{F} 127}=3 \% . T=37^{\circ} \mathrm{C}$. Left: HA12 $\Phi_{\mathrm{HA} 12}$ $=2 \%$. (x black) $\mathrm{NaCl} 0 \mathrm{M},(\mathrm{O}$ grey) $\mathrm{NaCl} 0.01 \mathrm{M}$, ( $\diamond$ blue) $\mathrm{KCl} 0.01 \mathrm{M}$, (O red) $\mathrm{NaBr} 0.01 \mathrm{M}$, ( $\diamond$ orange) $\mathrm{KBr} 0.01 \mathrm{M}$. Right: $\mathrm{HA} 300 \Phi_{\text {HA300 }}=2 \%$. (x black) NaCl $0 \mathrm{M}$, ( $\bigcirc$ grey) $\mathrm{NaCl} 0.01 \mathrm{M}$, ( $\diamond$ blue) $\mathrm{KCl} 0.01 \mathrm{M}$, ( $O$ red) $\mathrm{NaBr} 0.01 \mathrm{M}$, ( $\diamond$ orange) $\mathrm{KBr} 0.01 \mathrm{M}$. Full lines correspond to the best fits of the scattering data.
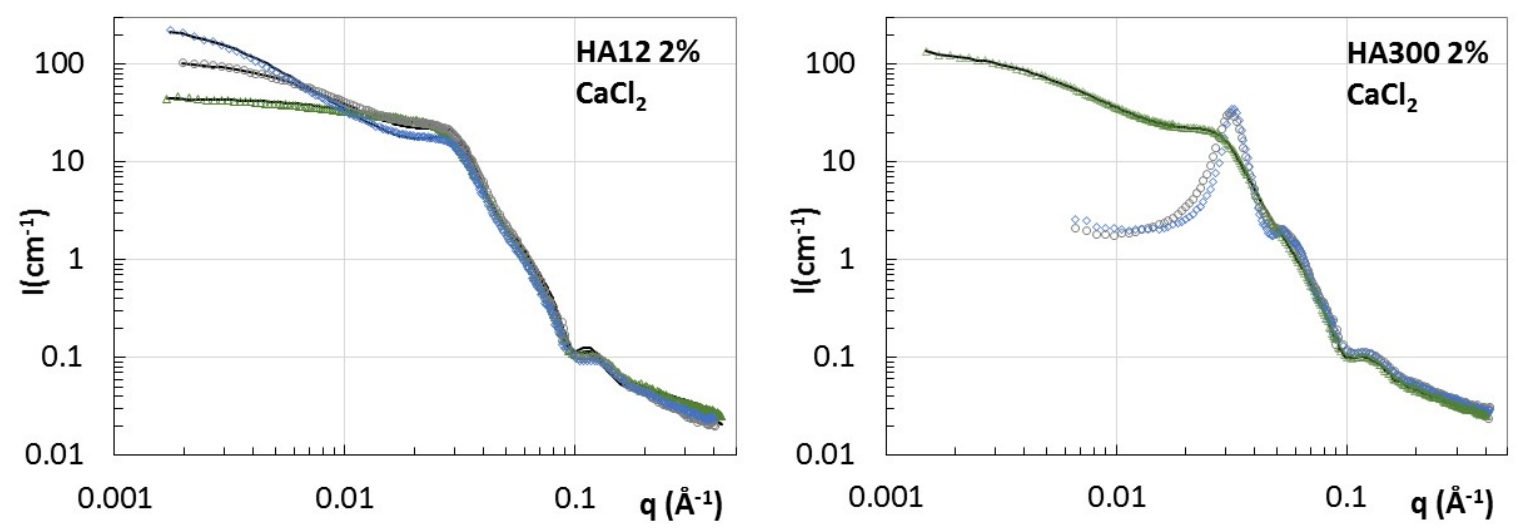

Figure 7: SANS data showing the effect of $\mathrm{CaCl}_{2} . \Phi_{\mathrm{F} 127}=3 \%$. $\mathrm{T}=37^{\circ} \mathrm{C}$. Left: $\mathrm{HA} 12 \Phi_{\mathrm{HA} 12}=2 \%$. ( $\triangle$ green) $\mathrm{CaCl}_{2} 0.033 \mathrm{M}$, (O grey) $\mathrm{CaCl}_{2} 0.05 \mathrm{M}$, ( $\diamond$ blue) $\mathrm{CaCl}_{2} 0.1 \mathrm{M}$. Right: $\mathrm{HA} 300 \Phi_{\text {HA300 }}=2 \%$. ( $\triangle$ green) $\mathrm{CaCl}_{2}$ $0.033 \mathrm{M}$, (O grey) $\mathrm{CaCl}_{2} 0.05 \mathrm{M}$, ( $\diamond$ blue) $\mathrm{CaCl}_{2} 0.1 \mathrm{M}$. Full lines correspond to the best fits of the scattering data before crystallization.

Table 7: $\mathrm{F} 127 \Phi_{\mathrm{F} 127}=3 \%, \mathrm{HA} 12 \Phi_{\mathrm{HA}}=2 \%, \mathrm{~T}=37^{\circ} \mathrm{C}$. Effect of the nature of the salt. Fit parameters to a core-shell form factor model with a square-well potential for the structure factor. Same parameter definition as in Table 3.

\begin{tabular}{|c|c|c|c|c|c|c|c|}
\hline salt & $\mathrm{NaCl}$ & $\mathrm{KCl}$ & $\mathrm{NaBr}$ & $\mathrm{KBr}$ & $\mathrm{CaCl}_{2}$ & $\mathrm{CaCl}_{2}$ & $\mathrm{CaCl}_{2}$ \\
\hline$\Phi_{\mathrm{HA}}$ & 0.02 & 0.02 & 0.02 & 0.02 & 0.02 & 0.02 & 0.02 \\
\hline $\mathrm{C}_{\text {salt }}(\mathrm{mol} / \mathrm{L})$ & 0.1 & 0.1 & 0.1 & 0.1 & 0.033 & 0.05 & 0.1 \\
\hline$\Phi_{\mathrm{m}}$ & 0.10 & 0.10 & 0.10 & 0.10 & 0.10 & 0.10 & 0.10 \\
\hline $\operatorname{Rc}(\AA)$ & 45 & 45 & 45 & 45 & 45 & 45 & 45 \\
\hline$e_{\text {sh }}(\AA)$ & 60 & 60 & 60 & 60 & 60 & 60 & 60 \\
\hline$\rho_{c}\left(10^{-6} \AA^{-2}\right)$ & 0.4 & 0.4 & 0.4 & 0.4 & 0.4 & 0.4 & 0.4 \\
\hline$\rho_{s h}\left(10^{-6} \AA^{-2}\right)$ & 5.8 & 5.8 & 5.8 & 5.8 & 5.8 & 5.8 & 5.8 \\
\hline$\rho_{\text {solv }}\left(10^{-6} \AA^{-2}\right)$ & 6.4 & 6.4 & 6.4 & 6.4 & 6.4 & 6.4 & 6.3 \\
\hline$D_{m}(\AA)$ & 217 & 222 & 210 & 207 & 214 & 202 & 202 \\
\hline $\mathrm{N}_{\text {mic }}$ & 1 & 1 & 3 & 2 & 4 & 15 & 45 \\
\hline$\xi(\AA)$ & 60 & 60 & 60 & 60 & 60 & 60 & 60 \\
\hline $\mathrm{U}_{0}\left(\mathrm{k}_{\mathrm{B}} \mathrm{T}\right)$ & 0.6 & 0.55 & 0.7 & 0.7 & 0.9 & 1.85 & 2.29 \\
\hline
\end{tabular}


Table 8: $\mathrm{F} 127 \Phi_{\mathrm{F} 127}=3 \%, \mathrm{HA} 300 \Phi_{\mathrm{HA}}=2 \%, \mathrm{~T}=37^{\circ} \mathrm{C}$. Effect of the nature of the salt. Fit parameters to a coreshell form factor model and a square-well potential for the structure factor. Same parameter definition as in Table 3.

\begin{tabular}{l|ccccc} 
salt & $\mathrm{NaCl}$ & $\mathbf{K C l}$ & $\mathbf{N a B r}$ & $\mathbf{K B r}$ & $\mathbf{C a C l}_{\mathbf{2}}$ \\
\hline$\Phi_{\text {HA }}$ & 0.02 & 0.02 & 0.02 & 0.02 & 0.02 \\
$\mathrm{C}_{\text {salt }}(\mathrm{mol} / \mathrm{L})$ & 0.1 & 0.1 & 0.1 & 0.1 & 0.033 \\
$\Phi_{\mathrm{m}}$ & 0.11 & 0.10 & 0.10 & 0.10 & 0.10 \\
$\operatorname{Rc}(\AA)$ & 45 & 45 & 45 & 45 & 45 \\
$\mathrm{e}_{\text {sh }}(\AA)$ & 60 & 60 & 60 & 60 & 60 \\
$\rho_{\mathrm{c}}\left(10^{-6} \AA^{-2}\right)$ & 0.4 & 0.4 & 0.4 & 0.4 & 0.4 \\
$\rho_{\text {sh }}\left(10^{-6} \AA^{-2}\right)$ & 5.8 & 5.8 & 5.8 & 5.8 & 5.8 \\
$\rho_{\text {solv }}\left(10^{-6} \AA^{-2}\right)$ & 6.4 & 6.4 & 6.4 & 6.4 & 6.4 \\
$D_{m}(\AA)$ & 209 & 208 & 203 & 202 & 202 \\
$\mathrm{~N}_{\text {mic }}$ & 6 & 5 & 15 & 15 & 31 \\
$\xi(\AA)$ & 60 & 60 & 60 & 60 & 60 \\
$\mathrm{U}_{0}\left(\mathrm{k}_{\mathrm{B}} \mathrm{T}\right)$ & 1.35 & 1.23 & 1.8 & 1.78 & 1.95
\end{tabular}

Influence of the size of the Pluronic micelles

Pluronics form a wide group of PEO-PPO-PEO triblock copolymers and offer the possibility to tune easily the size of the micelle by keeping the same chemical characteristics. P123 was then chosen to keep the same number of PO units for the core but has a smaller shell with $30 \mathrm{EO}$ units. The scattering of P123 with HA12 and HA300 at different volume fractions is presented in Figure SI 15 and fit parameters are given in Table SI 2. The SANS data analysis shows that the pure P123 micelles in brine solution have a total radius of $73 \AA$. As for F127, the radius of the core is $45 \AA$ but the hydrophilic shell thickness is smaller ( $28 \AA$ ) and the average of SLD of $2.10^{-6} \AA^{-2}$ indicates that the shell contains only $23 \%$ of water. We can note that at the Pluronic concentration of $3 \%$, the structure factor is close to the unity for P123 and the scattering curve reaches a plateau at $100 \mathrm{~cm}^{-1}$ whereas an interaction peak was clearly visible for F127.

As with F127, we observe for the lowest polyelectrolyte concentrations an increase of the scattering intensity at low q. The depth of the attractive potential is $1.2 \mathrm{k}_{\mathrm{B}} \mathrm{T}$ for $\mathrm{HA} 12$ at $\Phi_{\mathrm{HA}}=2 \%$ and $0.4 \mathrm{k}_{\mathrm{B}} \mathrm{T}$ for $\mathrm{HA} 300$ at $\Phi_{\mathrm{HA}}=1 \%$. With F127 at the same HA concentrations, we found $0.66 \mathrm{k}_{\mathrm{B}} \mathrm{T}$ (HA12) and $1 \mathrm{k}_{\mathrm{B}} \mathrm{T}$ (HA300). The appearance of the crystalline phase occurs at significantly lower HA volume fractions for P123 than for $\mathrm{F} 127$ i.e. $4 \%$ vs $7 \%$ with $\mathrm{HA} 12$ and $2 \%$ vs $4 \%$ with HA300.

\section{Discussion}

Colloids - polyelectrolytes systems exhibit a large variety of phase diagrams and structures due to a complex interplay between the different attractive (van der Waals, depletion, electrostatic) and repulsive forces (hydrophobic, steric, and electrostatic). Addition of salt modifies not only the ionic strength and the electrostatic interaction but also the water solvency and the hydrogen bonding.

For oppositely charged systems, the electrostatic interaction (and the release of counterions) is the motor of the complexation. The length of the polyelectrolyte chain, its concentration and the salinity 
play a role on the structure of the complexes. The phase diagram and the structure of the complexes formed by lysozyme and PSSNa have been investigated in [53]. It is shown that for a given [PSS] / [lysozyme] ratio, gels are formed with the long chains and dense packed globules are observed with short chains. However, the crossover between these two regimes can be tuned with the chain concentration or the ionic strength. SANS combined with contrast variation experiments evidenced the reduction of the persistence length of the polyelectrolyte chain in presence of protein in the gel phase and a more collapsed state of the polyions in the globules. When PSS is replaced by HA, the semirigid nature of HA compared to that of PSS brings an additional constrain, hindering the chain wrapping around the colloids. Effectively, in the HA / lysozyme system, the formation of rod-like shape structures which extend on larger scales than the persistence length of HA is evidenced due to the electrostatic repulsion between neighboring proteins attached to the HA backbone [54]. Such behavior has been further generalized as a function of the ratio between the total persistence length of the polyelectrolyte and the colloids [50]. In addition to the electrostatic interaction, hydrogen bonds can play an important role in the structure and dynamics of hyaluronic gels [55] and in general in polyelectrolytes complexes. It has been shown that at low pH, F127 monomers can interact with the carboxylic group of polyacids. The more hydrophobic chains bind with the PPO blocks of F127 inducing drastic change in the critical micellar temperature, and the more hydrophilic wrap around the individual F127 micelles forming nanosized dispersed complexes [56].

Unlike the previously described studies, Pluronic are nonionic and none of the structural organizations described above fully corresponds to those found in the HA / F127 system. When the attractive electrostatic interaction is reduced new competing interactions are revealed. For non-adsorbing polymers a depletion effect can be observed leading to a macroscopic phase separation into colloidsrich and colloids-poor phases. This depletion interaction depends on the excluded volume that is controlled by the nature of the different constituents, their concentration and the solution conditions. Despite the importance of the depletion interaction for the phase behavior in colloidal dispersions and therefore, its role in industrial formulation and biology, only limited experimental structural studies on model systems have been carried out. In this context, scattering experiments provide a unique way to reveal the nature of the interactions prior to the phase separation. In particular, SANS combined with contrast matching can be used to reveal the colloids partial structure factor that is directly linked to the interaction potential.

Depletion effects in neutral particle / polymer model systems have been investigated by SANS [32, 5759]. First measurements were carried out on calcium carbonate particles $[57,58]$ stabilized with by an adsorbed surfactant layer in presence of polyethylene polypropylene copolymers in decane $\left(R_{\mathrm{G}} / R \approx 2\right)$. No macroscopic phase separation was observed but an increase of the scattered intensity was detected at low q. The colloids partial structure factor could be fitted using an attractive interaction potential developed by Vrij and valid for small polymer chains [11]. An effective attraction between colloids could be evidenced in presence of polymers. This depletion induced attraction was found to increase with polymer concentration and level off slightly above $c^{*}$.

Charged colloidal dispersion with neutral polymers shows a more complex phase diagram due to the repulsive interactions between particles [60-62]. The phase behavior now depends on the balance between the repulsive (colloids-colloids, polymer-polymer) and attractive (depletion) interactions. This subtle interplay can be varied by changing the electrolyte or the polymer concentration. SANS can link the phase diagram with the colloidal organization and the particle interaction under salt and polymer addition. Charged silica particles with poly(ethylene glycol) in water have been recently reported $(0.3$ $<R_{\mathrm{G}} / R<0.7, c<c^{*}$ ) [61-62]. In salt-free solutions, electrostatic repulsion between charged colloids 
stabilizes the dispersion: a single homogeneous phase is observed even at high polymer concentration. Addition of electrolyte screens the electrostatic and a depletion induced microscopic phase separation appears when increasing the polymer concentration (turbid solutions due to particle association). Further addition of polymers modifies the strength of the depletion attraction and a transition to a single homogenous phase is observed (reentrant phase). When poly(ethylene glycol) is replaced by block copolymers (Pluronic P85) [60], the depletion effect is enhanced when the temperature is increased due to the formation of spherical Pluronic micelles and the induced variation of the excluded volume.

As in previous studies, experimental work on depletion usually concerns nonionic polymers and neutral or charged colloids in dilute regime. Semi-dilute polymer solutions in which the mesh size fixes the interaction range are rarely considered. Furthermore, there is a lack of studies on polyelectrolytes although they form a very important class of water-soluble macromolecules and have many implications in biology and technology. The present study gives new experimental structural findings on depletion effects in charged polymers / neutral colloids using a simple model system based on linear polysaccharides and Pluronic micelles. HA / F127 mixtures in aqueous solution have been investigated in semidilute regime by DSC and SANS. We explored the role of different parameters such as the HA concentration (above $c^{*}$ ), the chain length, the nature and salt concentration as well as the size of the micelles (F127, P123) on the critical micellar temperature, the structural organization and the stability of the solutions.

The size ratio $Q$ characterizing our system is determined from the estimated radius of gyration of the polyions $R_{\mathrm{G}}$ and the radius of the micelles $R(R \approx 105 \AA)$. $Q$ is close to 1 for HA12 $\left(69<R_{\mathrm{G}}<92 \AA\right)$ and in between 5 to 21 for $\mathrm{H} 300$ ( $470 \AA<R_{\mathrm{G}}<2200 \AA$ ). We are in the protein limit for HA300 and in an intermediate situation for HA12. Mixtures are in semi-dilute regime for all investigated polymer concentrations (from 1 to $7 \%$ ). Only the lowest volume fraction (1\%) with HA12 is close to $c^{*}$. In this entangled regime the range of attraction of the depletion equals the correlation length $\xi$ of the polymer network. The organization of the solution should no longer be sensitive to the molar mass of the chains. $\xi$ varies with the polymer concentration and the ionic strength of the solutions. For salt concentration around $0.1 \mathrm{~mol} / \mathrm{L}$, the Debye screening length is of the order of $10 \AA$ i.e. much less that the intrinsic persistence length $L_{0}$ of polymer. Electrostatic interaction between charged monomers is screened and polyions can be treated as neutral chains in good solvent conditions. For salt-free and brine solutions $\left(c_{\text {salt }}>0.1 \mathrm{~mol} / \mathrm{L}\right), \xi$ only depends on the polymer concentration as given by eq. $3 . \mathrm{In}$ this system, the radius of the particles is larger than the correlation length (from 75 to $40 \AA$ ), the size ratio $Q$ now defined as $D / \xi$ lies in between 3 and 5 .

In this new polymer / colloid system, SANS intensity is dominated by the signal of the micelles. Scattering experiment gives a direct insight into the F127 partial structure factor, and therefore, to the form factor $\mathrm{P}(\mathrm{q})$ and the dispersion state of the Pluronic micelles $(\mathrm{S}(\mathrm{q}))$. SANS measurements are very sensitive to the salt addition and show substantial modifications for certain compositions. Within the investigated HA and salt concentrations, the size and shape of the micelles as well as the SLD of the PEO shell are not modified by the presence of polymers. These observations are proofs that there are neither specific interaction nor adsorption between $\mathrm{HA}$ and the micelles. This is a very important point since the absence of adsorption is a prerequisite for depletion effects. On the other hand, clear modifications are observed at low $q$. The increase of the intensity is the signature of the formation of small clusters of micelles in the solution. Structural modifications are therefore mainly related to the dispersion state of the colloids as expected for an attractive depletion interaction [59,61,62]. In order to reveal precisely the nature of structural arrangements, we introduced a structure factor $S(q)$ based 
on a sticky hard-sphere model [31] in which the range of attraction is directly linked to the depletion layer thickness i.e. the correlation length $\xi$ of the polymer network.

The main results of the study can be summarized as follow:

(1) the presence of HA modifies the critical micellar temperature of the Pluronic copolymers to the same extend as an increase of the ionic strength.

(2) In salt free solutions, micelles are homogeneously distributed in the system and not perturbed by the presence of HA.

(3) By increasing the ionic strength the micellar organization is clearly changed: we observe the formation of clusters that evolve to a face-centered cubic liquid crystalline phase at high salt concentration. This behavior is reinforced by increasing the HA concentration and the molecular weight of the polyelectrolyte chains. It announces a macroscopic phase separation for non-viscous systems (HA12).

(4) The nature of the salt plays also a role and divalent cations such as $\mathrm{Ca}^{2+}$ promote the clustering of the micelles and their crystallization.

Without salt, we observe a single homogeneous phase whatever the HA molar mass or concentration. This situation is very similar to the symmetrical system where charged colloids are mixed with neutral polymers $[61,62]$ and stabilize the solutions. There are strong electrostatic repulsions between the semirigid polyions. A single phase in which the micelles are homogeneously distributed in the HA network is energetically more favorable than a phase separation that would decrease the available volume for the polyelectrolyte, reducing the distances between the charged chains.

In presence of salt $(0.1 \mathrm{M} \mathrm{NaCl}$ solution), the repulsive inter and intra chain repulsions are screened, the chains can get closer without a high energy cost. In addition, salt induces a change of the conformation of the polyelectrolyte chains and a reduction of the correlation length which in turn enhances the excluded volume for the micelles and induces an attractive depletion force. The micelles form first clusters which get bigger as the HA concentration increases. The intermicellar distance $D_{\mathrm{m}}$ is reduced until the crystallization occurs. Due to the high viscosity of the solutions, mixtures are turbid, but no macroscopic phase separation is observed. This general scenario is depicted in Figure 8 . The structural organization and the formation of finite size micellar clusters is similar that found in neutral calcium carbonate particles / polyethylene polypropylene copolymers systems $[57,58]$ or in charged silica particles / poly(ethylene glycol) $[61,62]$ except that no real crystallization (into a well ordered lattice) was observed in these systems.

How can we understand the enhanced clustering as the ionic strength increases above 0.1 M? For HA chains, at a salt concentration of $0.1 \mathrm{M}$, the electrostatic persistence length $\mathrm{L}_{\mathrm{e}}$ is reduced to $1.5 \AA$ [46] and the total chain stiffness is only mainly due to the intrinsic stiffness of the chain $\mathrm{L}_{0}$. The folding of the coil is mostly complete [52]. The enhanced clustering of the micelles observed above $0.1 \mathrm{M}$ is not due to the increase of excluded volume but to the weakening of the steric repulsion between the micelles. If salt affects the nonionic species in a lesser extent than charged colloids, it has nevertheless an influence on the Pluronic, both on the PO and EO groups, through solvation competition. On one side, the decrease of the critical micellar temperature in presence of salt is due to a reduced hydration of the PO groups and that all the more that the salt is cosmotropic. One the other side, the salt affects the hydrogen bonds between PEO and water and modify the PEO solubility. The differences observed between the different salts can be related to the Hofmeister classification [63-65]. At low salt concentration, it has been shown that subtle changes of the PEO layer coating silica particles, drive attraction [66,67]. Similarly, in our system, the weakening of the PEO layer and thus of the steric repulsion, is at the origin of the enhanced formation of micellar clusters when the ionic strength 
increases. Nevertheless, at a given ionic, the differences between the different salts cannot been fully rationalized. Larger clusters are formed in presence of $\mathrm{NaBr}, \mathrm{KBr}$ and $\mathrm{CaCl}_{2}$. Compare to $\mathrm{Na}^{+}, \mathrm{Br}^{+}$is more chaotropic but $\mathrm{Ca}^{2+}$ is more cosmotropic. More systematic investigations would be then necessary to clarify the effect of the nature of the ions.

An important point concerns the role of the HA molar weight on the organization and the stability of the solutions. At a given HA volume fraction, larger clusters are formed with the largest HA chain which also induces the crystallization of the micelles at a lower HA concentration. Similar effects are found when increasing the ionic strength. However, since we are in semi-dilute regime, attractive depletion interaction should no longer be dependent on the molar mass of the polymer chains as long as the solvent quality is preserved. For a given salt and HA concentration, the attractive interaction between the micelles is apparently larger for HA 300 than for HA12 while $\xi$ is identical. We believe that this unexpected effect is related to the low molar weight of $\mathrm{HA12}$ and its high polydispersity (PDI = 1.6). In this system, we are in an intermediate situation in between the colloidal and protein limits $\left(0.7<R_{G} / R\right.$ $<0.9$ ). The average extended length of the chains only corresponds to 3 or 4 intrinsic persistence lengths $L_{0}$ Due to the large polydispersity, very short chains (extended length close to 1 or $2 L_{0}$ ) cohabit with longer ones. In this inhomogeneous polymer solution, shorter chains are no longer flexible and do not show any conformational variation under salt addition. Their contribution to the excluded volume is negligible and the phase boundary is displaced to higher polymer concentration.

Finally, in a depletion process, the attractive strength is related to the size ratio between the species. The P123 micelles being smaller than the F127 micelles, it was expected that their crystallization occurs at larger volume fraction [16], but we observed the inverse trend. P123 micelles have a smaller PEO shell than F127 micelles. As for the salt effect, the steric repulsion between them is reduced and the crystallization occur at a lower depletion attraction. It is anticipated that a temperature increase reducing the water solvation and thus the size of the PEO shell, will also promote the clustering.

A rigorous and quantitative data analysis of the HA / Pluronic scattering would require the calculation of a dedicated potential of interaction between the two species which is above the scope of the paper. Asakura-Oosawa potential $[10,11]$ and models derived from it $[12,68]$ would certainly better reproduce the physics of the system. We are aware that the square-well potential chosen here to model the attractive depletion interaction is very raw. However, it gives a good agreement with the experimental neutron data and has already been used in several studies to model the depletion attraction [69]. One principal limitation is the impossibility to decouple numerically the width from the depth of the potential. To fix the width, the mesh size is needed but it is unknown in presence of micelles. Colloids and polymer exert a mutual influence. The impact of polymer on the colloids is easy to proof but the reverse is more subtle. With synthetic deuterated polymer, SANS in contrast matching conditions has allowed to extract the chain conformation close to the liquid-liquid phase boundary showing that colloids induce a significant shrinking (up to $50 \%$ ) of the polymer chains $[18,19,53]$. The chain behavior of natural polyelectrolytes in presence of colloids is still unknown and certainly deserve to be explored. 


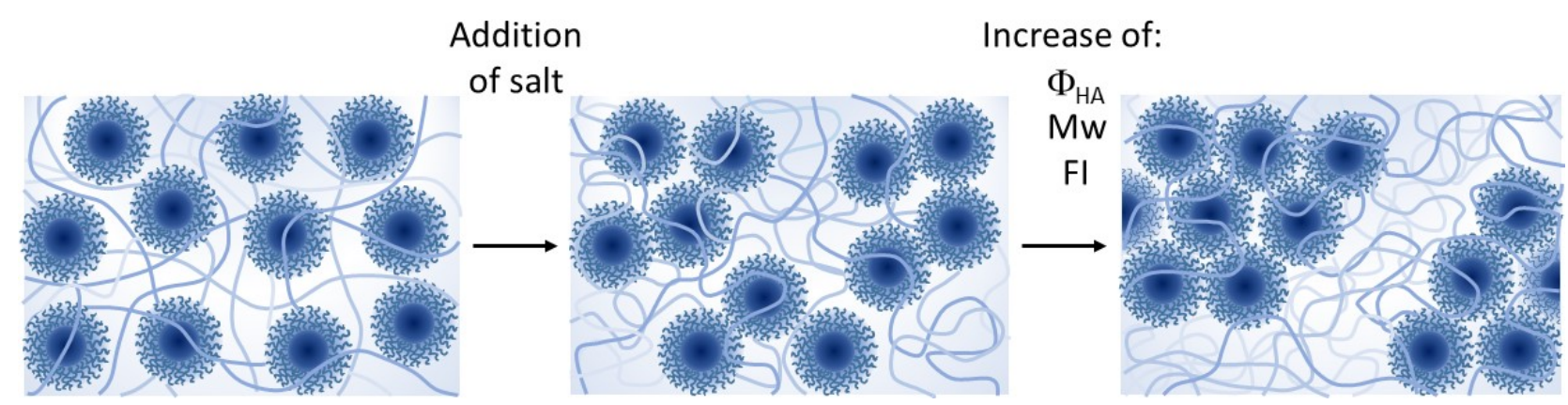

Figure 8: Schematic representation of the Pluronic micellar organization in presence of HA. The relative size between the micelles and the polymer network tries to respect the real organization in HA300 / F127 mixtures.

\section{Conclusion and perspective}

The model system composed of F127 Pluronic micelles and HA illustrates the well-known phenomenon that long range correlations in polymer solution induce an effective attraction between colloids that are immersed in the solution $[13,14]$. Because the polymer correlations depend not only on intrinsic polymer characteristics (mass, charge, rigidity,...) but also on environmental parameters that affect the chain solvency (temperature, ionic strength, nature of the solvent,...), the spatial organization and interaction between the colloids can be modified accordingly as evidenced by SANS. Addition of salt screens the electrostatic repulsion between the chains and induces a conformational change of the HA chain that increases the excluded volume. The micelles form first clusters with a finite size and then crystallize when the HA concentration increases. Although nonionic, Pluronic and their PEO moieties are also affected by the salt. The dehydration of the hydrophilic layer weakens the steric repulsion and also promotes the formation of clusters. Due to the possibility to easily vary either the ionic strength, the mass of the HA chain or the size of the Pluronic micelles, this system could be largely investigated to confront the existing theories with experiments to improve the understanding of the depletion attraction in the so-called "protein limit". In the medical field, macromolecular crowding and related effects due to excluded volume and increase of the viscosity, are at the origin of protein aggregation and fibrillation observed for example in Parkinson or Alzheimer diseases [70]. In consequence, disequilibrium of the blood saline composition could play a role in the formation of protein aggregates. Finally, the F127 / HA system appears as a good candidate to encapsulate active molecules to obtained a biocompatible solution for a local application. The incorporation of the linalool molecule in this system will be the topic of a forthcoming paper.

Acknowledgement: The authors thank the ILL for the beam time on the D33 instrument and the Partnership for Soft Condensed Matter (PSCM) for the sample preparation and complementary measurements (DSC). The neutron data set are available at doi:10.5291/ILL-DATA.9-12-417 and doi:10.5291/ILL-DATA.9-13-564 [71]. IG is very grateful to A. Brûlet and to the Laboratoire Léon Brillouin (LLB, Saclay, France) for the sabbatical period during when the first SANS test measurements were done. We are also grateful to the LLB for the beam time on PAXY and A. Brûlet, A. Chennevière and F. Cousin are thanked for their help on the instrument and data reduction. Xray measurements were performed on the beam line D2AM at the ESRF. Prof. Th. Zemb is gratefully acknowledged for his comments to clarify the presentation and helpful suggestions for the future research routes. Isabelle Morfin and Jérôme Combet express their fond acknowledgement to their colleague and friend Isabelle Grillo, recently deceased, who initiated this work. 
Conflict of interest: There is no conflict to declare.

Supporting Info

SANS data analysis; DSC Complementary Information; SANS Structural Characterization complementary information

\section{List of abbreviations:}

HA: Hyaluronic Acid

PEO: Poly(Ethylene Oxide)

PPO: Poly(Propylene Oxide)

DSC: Differential Scanning Calorimetry

SANS: Small Angle Neutron Scattering

SLD: Scattering Length Density 


\section{References}

1 A. V. Kabanova, E. V. Batrakova, V. Y. Alakhov, Advanced Drug Delivery Reviews, (2002), 54,759-779; Pluronic block copolymers for overcoming drug resistance in cancer.

2 K. S. Rankin and D. Frankel Soft Matter, Soft Matter (2016), 12, 3841-3848; Hyaluronan in cancer from the naked mole rat to nanoparticle therapy.

3 Y.-Y. Chen, H.-C. Wu, J.-S. Sun, G.-C. Dong, T.-W. Wang, Langmuir, (2013), 29, 3721-3729; Injectable and Thermoresponsive Self-Assembled Nanocomposite Hydrogel for Long-Term Anticancer Drug Delivery.

4 L. Mayol, F. Quaglia, A. Borzacchiello, L. Ambrosio, M. I. La Rotonda, European Journal of Pharmaceutics and Biopharmaceutics, (2008), 70, 199-206; A novel poloxamers/hyaluronic acid in situ forming hydrogel for drug delivery: Rheological, muco adhesive and in vitro release properties.

5 L. Mayol, M. Biondi, F. Quaglia, S. Fusco, A. Borzacchiello, L. Ambrosio, M. I. La Rotonda, Biomacromolecules, (2011), 12, 28-33; Injectable Thermally Responsive Mucoadhesive Gel for Sustained Protein Delivery.

6 Y-S. Jung , W. Park, H. Park, D.-K. Leeb, K. Na, Carbohydrate Polymers, (2017), 156, 403-408; Thermosensitive injectable hydrogel based on the physical mixing of hyaluronic acid and Pluronic F-127 for sustained NSAID delivery.

7 D. Seol, M. J. Magnetta, P. S. Ramakrishnan, G. L. Kurriger, H. Choe, K. Jang, J. A. Martin, T.-H. Lim, J Biomed Mater Res Part B (2013), 101B, 1508-1515; Biocompatibility and preclinical feasibility tests of a temperature-sensitive hydrogel for the purpose of surgical wound pain control and cartilage repair. 8 F. Wang, L. Li, B. Liu, Z. Chen, C. Li, Biomedicine and Pharmacotherapy, (2017), 86, 595-604;

Hyaluronic acid decorated pluronic P85 solid lipid nanoparticles as a potential carrier to overcome multidrug resistance in cervical and breast cancer.

9 D.D. Gurav, A. S. Kulkarni, A. Khan, V. S. Shinde, Colloids and Surfaces B: Biointerfaces, (2016), 143, 352-358; pH-responsive targeted and controlled doxorubicin delivery using hyaluronic acid nanocarriers.

10 S. Asakura, F. Oosawa, J. Chem. Phys., (1954), 22, 1255 - 1256; On interaction between 2 bodies immersed in a solution of macromolecules.

11 S. Asakura, F. Oosawa, J. Polymer Sc., (1958), 33, 183-192; Interaction between particles in solutions of macromolecules.

12 A. Vrij, Pure Appl. Chem., (1976), 48, 471-483; Polymers at Interfaces and the Interactions in Colloidal Dispersions.

13 P.G. de Gennes, Scaling concepts in polymer physics ; Cornell University Press, Ithaka, NY, 1979

14 R.P. Sear, Eur. Phys. J. B (1998), 1, 313-317; Scattering from small colloidal particles in a semidilute polymer solution.

15 R.P. Sear, Phys. Rev. Lett (2001), 86, 4696-4699; Phase separation in mixtures of colloids and long ideal polymer coils.

16 R.P. Sear, Phys. Rev. E (1997), 56, 4463-4466; Entropy-driven phase separation in mixtures of small colloidal particles and semidilute polymers.

17 W. K. Lim, A. R. Denton, J. Chem. Phys., (2014), 141, 114909:1-10; Polymer crowding and shape distributions in polymer-nanoparticle mixtures.

18 T. Kramer, R. Schweins, K. Huber, Macromolecules, (2005), 38, 9783-9793; Coil Dimensions of Polystyrene Chains in Colloid-Polymer Mixtures at the Protein Limit: A SANS Study.

19 C. Le Coeur, B. Demé, S. Longeville, Phys. Rev. E, (2009), 79, 031910- 1:4; Compression of random coils due to macromolecular crowding.

20 M. Fuchs, K.S. Schweizer, Europhys. Lett.,(2000), 51, 621-627; Structure and thermodynamics of colloid-polymer mixtures: A macromolecular approach.

21 M. Fuchs, K.S. Schweizer J. Phys.: Condens. Matter (2002), 14, R239-R269; Structure of colloidpolymer suspensions.

22 P.G. Bolhuis, E.J. Meijer, A.A. Louis, Phys.Rev. Lett. (2003), 90, 068304; Colloid-polymer mixtures in the protein limit. 
23 H.N.W. Lekkerkerker, W.C.K. Poon, P.N. Pusey, A. Stroobants, P.B. Warren, Europhys. Lett., (1992), 20, 559-564; Phase-behavior of colloid plus polymer mixtures.

24 G.J. Fleer, R. Tuinier Phys.Rev. E (2007), 76, 041802; Analytical phase diagram for colloid-polymer mixtures.

25 G.J. Fleer, R. Tuinier Adv. Colloid Interface Sci (2008), 143, 1-47; Analytical phase diagrams for colloids and non-adsorbing polymer.

26 C. Sommer, J. S. Pedersen, P. C. Stein, J. Phys. Chem. B (2004), 108, 6242-6249; Apparent Specific Volume Measurements of Poly(ethylene oxide), Poly(butylene oxide), Poly(propylene oxide), and Octadecyl Chains in the Micellar State as a Function of Temperature.

27 E. Buhler, F. Boué, Macromolecules (2004), 37, 1600-1610; Chain persistence length and structure in hyaluronan solutions : ionic strength dependence for a model semirigid polyelectrolyte.

28 E. Hecht, H. Hoffmann, Colloids Surface A: Physicochem. Eng. Aspects, (1995), 96, 181-197; Kinetic and calorimetric investigations on micelle formation of block copolymers of the poloxamer type. 29 https://www.ill.eu/instruments-support/computing-for-science/cs-software/all-software/lamp/ 30 I. Grillo, I. Morfin, S. Prévost, Langmuir, (2018), 34, 13395-13408; Structural Characterization of Pluronic Micelles Swollen with Perfume Molecules.

31 R.J. Baxter, J. Chem. Phys. (1968), 49, 2770; Percus-Yevick equation for hard sphere with surface adhesion.

32 R. Tuinier, E. ten Grotenhuis, C. Holt, P.A. Timmins, C.G. de Kruif, PRE, (1999), 60, 848-856; Depletion interaction of casein micelles and an exocellular polysaccharide.

33 M. Sztucki, T. Narayanan, G. Belina, A. Moussaïd, F. Pignon, H. Hoekstra, PRE, (2006); 74, 051504:111 , Kinetic arrest and glass-glass transition in short-ranged attractive colloids

34 G. Wanka, H. Hoffmann, W. Ulbricht, Macromolecules (1994), 27, 4145-4159; Phase diagrams and aggregation behavior of poly (oxyethylene)-poly (oxypropylene) -poly( oxyethylene) triblock copolymers in aqueous solutions.

35 P. Alexandridis, T. A. Hatton, Colloids and Surfaces A: Physicochemical and Engineering Aspects, (1995), 96, 1-46; Poly(ethylene oxide)-poly(propylene oxide )-poly (ethylene oxide) block copolymer surfactants in aqueous solutions and at interfaces: thermodynamics, structure, dynamics, and modeling.

36 N. Pandit, T. Trygstad, S. Croy, M. Bohorquez, C. Koch, JCIS (2000) 222, 213-220; Effect of salts on the micellization, clouding, and solubilization behavior of Pluronic F127 Solutions.

37 C.B. Anderson, S.M. Cox, A.V. Ambardekar, S. K. Mallapragada, J. Pharm. Sci. (2002), 91, 180- 188; The effect of salts on the micellization temperature of aqueous poly(ethylene oxide)-b-poly(propylene oxide)-b-poly(ethylene oxide) solutions and the dissolution rate and water diffusion coefficient in their corresponding gels.

38 J. S. Pedersen, M. C. Gerstenberg, Colloids and Surfaces A: Physicochem. Eng. Aspects, (2003), 213, 175-187; The structure of P85 Pluronic block copolymer micelles determined by small-angle neutron scattering

39 Manet S., Lecchi A., Impéror-Clerc M., Zholobenko V., Durand D., Oliveira C., Pedersen J.S., Grillo I., Meneau F, and Rochas C. J. Phys. Chem. B, (2011) 115, 11318-11329; Structure of Micelles of a Nonionic Block Copolymer Determined by SANS and SAXS.

40 Y. Kadam, R. Ganguly, M. Kumbhakar, V. K. Aswal, P. A. Hassan, P. Bahadur, J. Phys. Chem. B 2009, 113, 16296-16302; Time dependent sphere-to-rod growth of the pluronic micelles: Investigating the role of core and corona solvation in determining the micellar growth rate.

41 L. Fan, M. Degen, N. Grupido, S. Bendle, P. Pennartz, Materials Science and Engineering A, (2010), 528, 127-136; Effects of molecular weight, temperature and salt on the self-assembly of triblock copolymer solutions.

42 T.J. Odijk, . Polym. Sci., Polym. Phys. Ed. (1977), 15, 477-483; Polyelectrolytes near the rod limit. 43 J. Skolnick, M. Fixman, Macromolecules, (1977), 10, 944-948; Electrostatic Persistence Length of a Wormlike Polyelectrolyte.

44 M. Fixman, J. Chem. Phys., (1982), 76, 6346-6353; The flexibility of polyelectrolyte molecules. 
45 H. Benoit, P. Doty J. Phys. Chem. (1953), 57, 958-963; Light Scattering from Non-Gaussian Chains. 46 E. Buhler, F. Boué, Macromolecules (2004), 37, 1600-1610; Chain persistence length and structure in hyaluronan solutions : ionic strength dependence for a model semirigid polyelectrolyte.

47 K. Salamon, D. Aumiler, G. Pabst, T. Vuletić, Macromolecules (2013), 46, 1107-1118; Probing the Mesh Formed by the Semirigid Polyelectrolytes.

48 M. Nierlich, C.E. Williams, F. Boué, J.-P. Cotton, M. Daoud, B. Farnoux, G. Jannink, C. Picot, M. Moan, C. Wol, M. Rinaudo, P.-G.de Gennes, J Phys. (Fr.) (1979), 40, 701-704; Small angle neutron scattering by semi-dilute solutions of polyelectrolyte.

49 F. Bonnet, R. Schweins, F. Boué, E. Buhler, EPL, (2008), 83, 48002:1-5; Suppression of aggregation in natural-semiflexible/flexible polyanion mixtures, and direct check of the OSF model using SANS.

50 L. Shi, F. Carn, F. Boué, E. Buhler, PRE, (2016), 94, 032504:1-12; Role of the ratio of biopolyelectrolyte persistence length to nanoparticle size in the structural tuning of electrostatic complexes.

51 G. Wanka, H. Hoffmann, W. Ulbricht Macromolecules (1994), 27, 4145-4159; Phase diagrams and aggregation behavior of POE-POP-POE triblocks copolymers in aqueous solutions.

52 P. Gribbon, B. C. Heng, T. E. Hardingham, Biophysical Journal Volume 77 October 1999 2210-2216; The Molecular Basis of the Solution Properties of Hyaluronan Investigated by Confocal Fluorescence Recovery After Photobleaching.

53 J. Gummel, F. Cousin, F. Boué, Macromolecules (2008), 41, 2898-2907; Structure Transition in PSS/Lysozyme Complexes: A Chain-Conformation-Driven Process, as Directly Seen by Small Angle Neutron Scattering.

54 I. Morfin, E. Buhler, F. Cousin, I Grillo, F. Boué, Biomacromolecules (2008), 12, 859-870; Rodlike Complexes of a Polyelectrolyte (Hyaluronan) and a Protein (Lysozyme) Observed by SANS.

55 P. Bełdowski, P. Weber, A. Dédinaité, P. M. Claesson, A.Gadomski, Soft Matter, 2018, 14, 89979004; Physical crosslinking of hyaluronic acid in the presence of phospholipids in an aqueous nanoenvironment.

56 Y. Wanga, S. A. Sukhishvili, Soft Matter, 2016, 12, 8744-8754; Hydrogen-bonded polymer complexes and nanocages of weak polyacids templated by a Pluronics block copolymer.

57 X. Ye, T. Narayanan, P. Tong, J. S. Huang, M. Y. Lin, B. L. Carvalho, L. J. Fetters, Phys. Rev. E 54, 6500 (1996); Depletion interactions in colloid-polymer mixtures

58 X. Ye, T. Narayanan, P. Tong, J. S. Huang, Phys. Rev. Lett. 76, 4640 (1996); Neutron Scattering Study of Depletion Interactions in a Colloid-Polymer Mixture

59 K. J. Mutch, J. S. van Duijneveldt, J. Eastoe, I. Grillo, R. K. Heenan, Langmuir, (2009), 25, 3944-3952; Testing the Scaling Behavior of Microemulsion-Polymer Mixtures

60 S. Kumar, M.-J. Lee, V. K. Aswal, S.-M. Choi, Physical Review E, 2013, 87, 042315; Block-copolymerinduced long-range depletion interaction and clustering of silica nanoparticles in aqueous solution.

61 S. Kumar, D. Ray, V. K. Aswal, J. Kohlbrecher, Physical Review E, (2014), 90, 042316; Structure and interaction in the polymer-dependent reentrant phase behavior of a charged nanoparticle solution.

62 S. Kumar, V. K. Aswal, J. Kohlbrecher, Langmuir, (2016), 32, 1450; Small-Angle Neutron Scattering Study of Interplay of Attractive and Repulsive Interactions in Nanoparticle-Polymer System

63 M. Ataman, Colloid Polym. Sci., (1987), 265, 19-25; Properties of aqueous salt solutions of poly(ethylene oxide). Cloud points, $\theta$ temperatures.

64 M. Ataman, E.A. Boucher, J. Polym. Sci., Part B: Polym. Phys., (1982), 20, 1585-1592; Properties of aqueous salt-solutions of poly(ethylene oxide).

65 Y. Zhang, P.S. Cremer, Curr. Opin. Chem. Biol. (2006), 10, 658-663; Interactions between macromolecules and ions: The Hofmeister series.

66 M. Zackrisson, A. Stradner, P. Schurtenberger, J. Bergenholtz, Langmuir, (2005), 21, 10835-10845; Small-Angle Neutron Scattering on a Core-Shell Colloidal System: A Contrast-Variation Study.

67 M. Zackrisson Oskolkova, A. Stradner, J. Ulama, J. Bergenholtz, RSC Adv., 2015, 5, 25149-25155; Concentration-dependent effective attractions between PEGylated nanoparticles. 
68 T. D. Edwards, M. A. Bevan, Langmuir, (2012), 28, 13816-13823; Depletion-Mediated Potentials and Phase Behavior for Micelles, Macromolecules, Nanoparticles, and Hydrogel Particles.

69 A. Muratov, A. Moussaïd, T. Narayanan, E. I. Kats, J. Chem. Phys. (2009), 131, 054902:1-11; A Percus-Yevick description of the microstructure of short-range interacting metastable colloidal suspensions.

70 L. A. Munishkina, E. M. Cooper, V. N. Uversky, A. L. Fink, J. Mol. Recognit. 2004; 17: 456-464; The effect of macromolecular crowding on protein aggregation and amyloid fibril formation.

71 I. Grillo, J. Combet, I. Morfin, (2015). Formation of pluronic micellar clusters induced by the change of chain conformation of hyaluronic acid after addition of salt. Institut Laue-Langevin (ILL) doi:10.5291/ILL-DATA.9-12-417; I. Grillo, J. Combet, I. Morfin, (2014). Structural investigation of Hyaluronan / Pluronic aggregates: a possible system for drug delivery with specific targeting. Institut Laue-Langevin (ILL) doi:10.5291/ILL-DATA.9-13-564 